\title{
Molecular jets driven by high-mass protostars: a detailed study of the IRAS 20126+4104 jet ${ }^{\star}$
}

\author{
A. Caratti o Garatti ${ }^{1}$, D. Froebrich ${ }^{2}$, J. Eislöffel $^{1}$, T. Giannini ${ }^{3}$, and B. Nisini ${ }^{3}$ \\ 1 Thüringer Landessternwarte Tautenburg, Sternwarte 5, 07778 Tautenburg, Germany \\ e-mail: [caratti; jochen]@tls-tautenburg.de \\ 2 Centre for Astrophysics and Planetary Science, University of Kent, Canterbury, CT2 7NH, UK \\ e-mail: df@star.kent.ac.uk \\ 3 INAF - Osservatorio Astronomico di Roma, via Frascati 33, 00040 Monte Porzio, Italy \\ e-mail: [giannini;nisini]@oa-roma.inaf.it
}

Received 5 February 2008 / Accepted 13 March 2008

\begin{abstract}
Context. Protostellar jets from intermediate- and high-mass protostars provide an excellent opportunity to understand the mechanisms responsible for intermediate- and high-mass star-formation. A crucial question is if they are scaled-up versions of their low-mass counterparts. Such high-mass jets are relatively rare and, usually, they are distant and highly embedded in their parental clouds. The IRAS $20126+4104$ molecular jet, driven by a $10^{4} L_{\odot}$ protostar, represents a suitable target to investigate.

Aims. We present here an extensive analysis of this protostellar jet, deriving the kinematical, dynamical, and physical conditions of the $\mathrm{H}_{2}$ gas along the flow.

Methods. The jet was investigated by means of near-IR $\mathrm{H}_{2}$ and [Fe II] narrow-band imaging, high-resolution spectroscopy of the 1-0 S(1) line $(2.12 \mu \mathrm{m})$, NIR $(0.9-2.5 \mu \mathrm{m})$ low-resolution spectroscopy, along with ISO-SWS and LWS spectra (from 2.4 to $200 \mu \mathrm{m})$. Results. The flow shows a complex morphology. In addition to the large-scale jet precession presented in previous studies, we detect a small-scale wiggling close to the source, which may indicate the presence of a multiple system. The peak radial velocities of the $\mathrm{H}_{2}$ knots range from -42 to $-14 \mathrm{~km} \mathrm{~s}^{-1}$ in the blue lobe, and from -8 to $47 \mathrm{~km} \mathrm{~s}^{-1}$ in the red lobe. The low-resolution spectra are rich in $\mathrm{H}_{2}$ emission, and relatively faint [Fe II] (NIR), [O I] and [C II] (FIR) emission is observed in the region close to the source. $\mathrm{A}$ warm $\mathrm{H}_{2}$ gas component has an average excitation temperature that ranges between $2000 \mathrm{~K}$ and $2500 \mathrm{~K}$. Additionally, the ISO-SWS spectrum reveals a cold component $(520 \mathrm{~K})$ that strongly contributes to the radiative cooling of the flow and plays a major role in the dynamics of the flow. The estimated $L_{\mathrm{H}_{2}}$ of the jet is $8.2 \pm 0.7 L_{\odot}$, suggesting that IRAS 20126+4104 has a significantly increased accretion rate compared to low-mass YSOs. This is also supported by the derived mass flux rate from the $\mathrm{H}_{2}$ lines $\left(\dot{M}_{\text {out }}\left(\mathrm{H}_{2}\right) \sim 7.5 \times 10^{-4} M_{\odot} \mathrm{yr}^{-1}\right)$. The comparison between the $\mathrm{H}_{2}$ and the outflow parameters strongly indicates that the jet is driving the outflow, at least partially. As already found for low-mass protostellar jets, the measured $\mathrm{H}_{2}$ outflow luminosity is tightly related to the source bolometric luminosity. Conclusions. As for a few other intermediate- and high-mass protostellar jets in the literature, we conclude that IRAS 20126+4104 jet is a scaled-up version of low-mass protostellar counterparts.
\end{abstract}

Key words. stars: pre-main-sequence - infrared: ISM - ISM: jets and outflows - ISM: kinematics and dynamics - individual objects: IRAS 20126+4104

\section{Introduction}

Protostellar jets and outflows are a ubiquitous phenomenon among young stellar objects (YSOs) of different masses and luminosities (see e.g. Shepherd 2003). They are usually explained as a consequence of accretion from a disc around the protostar (see e.g. Pudritz \& Norman 1986; Camenzind 1990). This is particularly true for low - and, partially, for intermediate and high-mass YSOs up to $L_{\text {bol }} \sim 10^{4} L_{\odot}$ (or spectral type B0), where collimated outflows, often driven by protostellar jets, have been observed (Shepherd 2003). In contrast, no highly collimated outflow or circumstellar disc has been observed in highmass protostars exceeding $10^{5} L_{\odot}$ (or O-type stars, the spectral type and $L_{\mathrm{bol}}$ of the M17 disc silhouette are not clear yet) (see e.g. Arce et al. 2007; Zinnecker \& Yorke 2007), and the formation mechanism of these latter objects is still being debated. The observations are, however, strongly limited by the

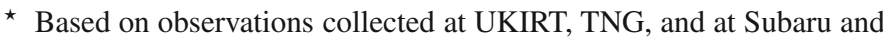
ISO data archive. large distance of the massive star-forming regions, the considerable extinction, and the short lifetime of massive YSOs. In addition, these objects are often grouped in small clusters, which confuses the morphology of massive star-forming regions even more. Therefore, optical and IR studies of intermediatehigh-mass protostellar jets are very rare, and only a few examples are present in the literature: e.g. IRAS 18162-2048 $\left(L_{\mathrm{bol}} \sim\right.$ $2 \times 10^{4} L_{\odot}$, Martí et al. 1995), IRAS $20126+4104\left(L_{\text {bol }} \sim 10^{4} L_{\odot}\right.$, Ayala et al. 1998), IRAS $16547-4247\left(6 \times 10^{4} L_{\odot}\right.$, Brooks et al. 2003), IRAS 18151-1208 $\left(2 \times 10^{4} L_{\odot}\right.$, Davis et al. 2004), and IRAS 11101-5829 (10 $L_{\odot}$, Gredel 2006), the M17 disc silhouette ( $M_{\text {YSO }} \sim 15 M_{\odot}$, Nürnberger et al. 2007). Expanding the number of observations of intermediate- and high-mass jets and comparing their general properties with those of lowmass protostellar jets is therefore important for understanding whether differences exist, or if high-mass protostellar jets are just scaled up versions of their low-mass counterparts. We therefore investigated the kinematical and physical properties of the IRAS $20126+4104$ jet by means of NIR narrow-band imaging, 
high-resolution and low-resolution IR spectroscopy. We then compared our findings with those of other high- and low-mass protostellar jets available in the literature.

IRAS $20126+4104$, at a distance $1.7 \mathrm{kpc}$, is a very well studied high-mass YSO $\left(M \sim 7 M_{\odot}\right.$, Cesaroni et al. 1997, 2005) in a very early stage of evolution. It is accreting mass at a very high rate $\left(\dot{M}_{\text {acc }} \sim 2 \times 10^{-3} M_{\odot} \mathrm{yr}^{-1}\right.$, Cesaroni et al. 2005) and it gives birth to a large poorly-collimated $\mathrm{CO}$ outflow. It harbours the first $\mathrm{H}_{2}$ jet detected from a high mass YSO (Ayala et al. 1998), which had previously been seen in $\mathrm{SiO}$ emission close to the source (Cesaroni et al. 1999). The $\mathrm{H}_{2}$ jet extends for about $1 \mathrm{pc}$. Its " $\mathrm{S}$ " shape morphology suggests that it is precessing with a period of $\sim 60000 \mathrm{yr}$ and with a wide precession angle of about $37^{\circ}$ (Shepherd et al. 2000). As a consequence, the inclination of the flow with respect to the plane of the sky changes strongly from $\sim 9^{\circ}$, close to the source, up to $\sim 45^{\circ}$ in the outer part of the flow.

The structure of this paper is as follows. In Sect. 2 our observations are presented. Section 3 reports an overview of our results, including the morphology of the $\mathrm{H}_{2}$ jet, its kinematics, the physical parameters of the gas, its energy, and mass flux rate. In Sect. 4 we briefly consider the cause of the newly detected small-scale precession mode. Then, we discuss our $\mathrm{H}_{2}$ data in relation to the $\mathrm{CO}$ outflow literature data. Finally, we compare the properties of the IRAS $20126+4104$ jet with other high- and low-mass protostellar jets.

\section{Observations and data reduction}

Our data were collected at the UK Infrared Telescope (UKIRT), and at the $3.5 \mathrm{~m}$ Italian Telescopio Nazionale Galileo (TNG). More data were retrieved from the Subaru and ISO archives ${ }^{1}$. The relevant information on the observational settings is summarised in Table 1.

\subsection{Imaging: $\mathrm{H}_{2}$ and [Fe II]}

We used narrow-band filters centred on the $\mathrm{H}_{2}(2.12 \mu \mathrm{m})$ and [Fe II] $(1.64 \mu \mathrm{m})$ lines to detect both molecular and ionic emission along the flow. Additional broad-band images $\left(H\right.$ and $\left.K^{\prime}\right)$ were gathered to remove the continuum. Our images were collected at UKIRT, using the near-IR instrument UIST (Ramsay Howat et al. 2004) ([Fe II], H), and at the TNG, using NICS (Baffa et al. 2001) $\left(\mathrm{H}_{2}, K^{\prime}\right)$. All the raw data were reduced using IRAF $^{2}$ packages, applying standard procedures for sky subtraction, dome flat-fielding, bad pixel and cosmic ray removal, and image-mosaicking. The resulting mosaics cover a region around IRAS $20126+4104$ of 3.4 $\times 3.4$ for UIST and $5: 6 \times 5$ ' 6 for NICS. The calibration for both instruments was obtained by means of photometric standard stars observed in both narrow- and broad-band filters. In the calibrated and continuumsubtracted narrow-band images, we measured $\mathrm{H}_{2}$ and [Fe II] fluxes for each detected knot using the task polyphot in IRAF, defining each region within a $3 \sigma$ contour level above the sky background.

Additional high-resolution, narrow-band images $\left(\mathrm{H}_{2}, \mathrm{Br}_{\gamma}\right.$, and $\left.K_{\text {cont }}\right)$ of the central part of the molecular flow $\left(\sim 35^{\prime \prime} \times 35^{\prime \prime}\right)$

\footnotetext{
1 These data are available at http://smoka.nao.ac.jp/ (Subaru), and http://www.iso.vilspa.esa.es/ida/index.html (ISO).

${ }^{2}$ IRAF (Image Reduction and Analysis Facility) is distributed by the National Optical Astronomy Observatories, which are operated by AURA, Inc., cooperative agreement with the National Science Foundation.
}

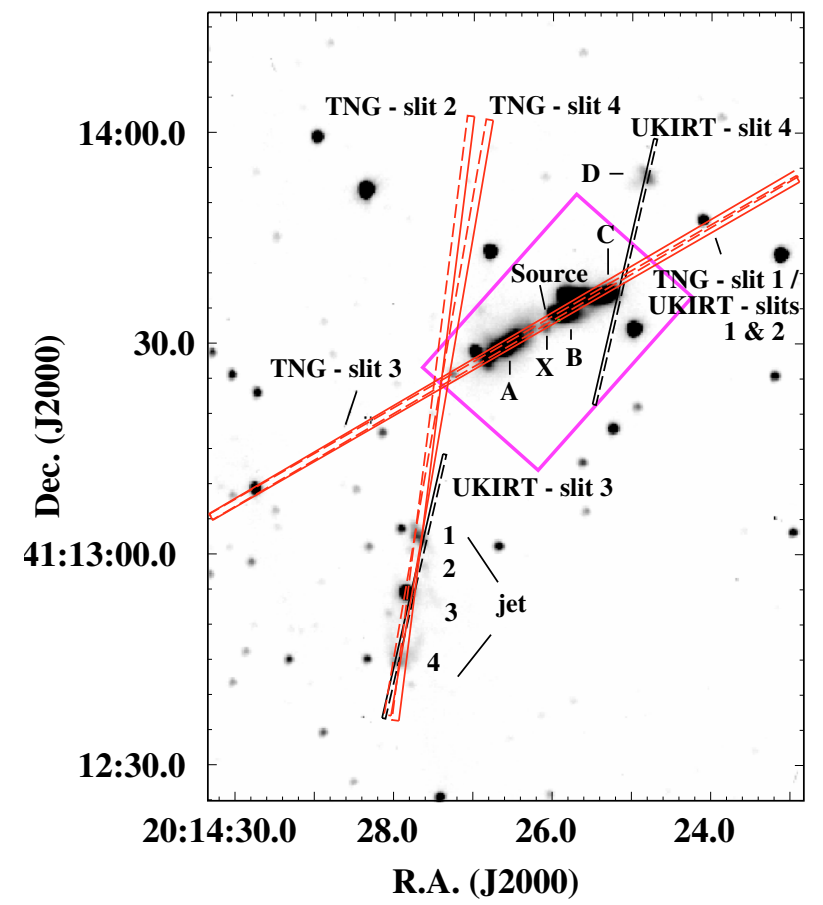

Fig. 1. Close up of the TNG $\mathrm{H}_{2}$ mosaic, showing the entire flow with superimposed positions of the slits and the ISO-SWS FoV. The positions of the $\mathrm{H}_{2}$ knots and the IRAS source $\left(\alpha=20^{\mathrm{h}} 14^{\mathrm{m}} 26.03^{\mathrm{s}}\right.$ and $\delta=41^{\circ} 13^{\prime} 32^{\prime \prime} .58$ [J2000], Hofner et al. 2007) are also indicated.

were retrieved from the Subaru data archive (Baba et al. 2002). The set of data were taken with the Coronagraphic Imager with Adaptive Optics (CIAO) (Murakawa et al. 2003), used as a highresolution NIR imager (0.'022/pixel). These data were used for a morphological study of the flow only close to the source (see Sects. 3.1 and 4.1).

Finally, we used $K$-band stars from the Two Micron All Sky Survey (2MASS) for the astrometric calibration of all the images. As a result the formal errors for the plate solutions are $\sim 0.5,0.4$, and 0.2 , for the NICS, UIST, and CIAO images, respectively.

\subsection{Spectroscopy}

\subsubsection{Low-resolution spectroscopy}

The NIR low-resolution spectroscopy ( $\mathcal{R} \sim 500$, slit width $1^{\prime \prime}$ ) was acquired with NICS during two runs at the TNG, using three different grisms covering all the full NIR spectrum $(0.9-2.5 \mu \mathrm{m})$ (see Table 1). The position of the slits is shown in Fig. 1. Slits 1 and 3 are positioned on the knots close to the source (knots A, $\mathrm{B}$, and C) and parallel to the jet axis, covering almost all the $\mathrm{H}_{2}$ emitting area. Slits 2 and 4 encompass the so-called "jet" (see Ayala et al. 1998), that is the farthest $\mathrm{H}_{2}$ emission in the red lobe (see also Fig. 1).

To perform our spectroscopic measurements, we adopted the usual $\mathrm{ABB}^{\prime} \mathrm{A}^{\prime}$ configuration, with a total integration time between $1800 \mathrm{~s}$ and $2400 \mathrm{~s}$ per grism per slit. Each observation was flat fielded, sky subtracted and corrected for the curvature caused by longslit spectroscopy, while atmospheric features were removed by dividing each spectrum by a telluric standard star (spectral $\mathrm{O}$ type). The wavelength calibration was obtained from Xenon and Argon lamps. The flux was calibrated observing the photometric standard star AS 35-0 (Hunt et al. 1998). As the 
Table 1. Journal of observations.

\begin{tabular}{cccccc}
\hline $\begin{array}{c}\text { IMate of obs. } \\
(\mathrm{d}, \mathrm{m}, \mathrm{y})\end{array}$ & $\begin{array}{c}\text { Telescope/ } \\
\text { Instrument }\end{array}$ & $\begin{array}{c}\text { Filter } \\
\text { Band }\end{array}$ & $\begin{array}{c}\text { Resolution } \\
\left({ }^{\prime \prime} / \text { pixel }\right)\end{array}$ & $\begin{array}{c}\text { Seeing } \\
\left({ }^{\prime \prime}\right)\end{array}$ & $\begin{array}{c}\text { Exp. time } \\
(\mathrm{s})\end{array}$ \\
\hline 01.10 .2006 & UKIRT/UIST & {$[\mathrm{Fe}$ II $]$} & 0.12 & 0.6 & 720 \\
01.10 .2006 & UKIRT/UIST & $H$ & 0.12 & 0.6 & 360 \\
06.08 .2006 & TNG/NICS & $\mathrm{H}_{2}$ & 0.25 & 1.2 & 900 \\
06.08 .2006 & TNG/NICS & $K^{\prime}$ & 0.25 & 1.2 & 90 \\
10.07 .2003 & Subaru/CIAO & $\mathrm{H}_{2}, \mathrm{Br} \gamma, K_{\text {cont }}$ & 0.022 & 0.9 & 1800 \\
\hline
\end{tabular}

SPECTROSCOPY

\begin{tabular}{|c|c|c|c|c|c|c|c|c|}
\hline $\begin{array}{l}\begin{array}{c}\text { Date of obs } \\
\text { (d.m.y) }\end{array} \\
\end{array}$ & $\begin{array}{l}\text { Telescope/ } \\
\text { Instrument }\end{array}$ & $\begin{array}{c}\text { Wavelength } \\
(\mu \mathrm{m})\end{array}$ & $\begin{array}{l}t_{\text {int }} \\
(\mathrm{s})\end{array}$ & $\begin{array}{l}\mathrm{PA} \\
\left(^{\circ}\right)\end{array}$ & $\begin{array}{c}\text { Slit/Aperture width } \\
\left({ }^{\prime \prime}\right)\end{array}$ & $\overline{\mathcal{R}}$ & "Encompassed knots & Notes \\
\hline 01.10 .2006 & UKIRT/CGS4 & 2.1218 & 2700 & 300 & 0.5 & 18500 & $\mathrm{~A} 1, \mathrm{~A} 2, \mathrm{X}, \mathrm{B}, \mathrm{C} 2$ & UKIRT-Slit1 \\
\hline 01.10 .2006 & UKIRT/CGS4 & 2.1218 & 2700 & 300 & 0.5 & 18500 & $\mathrm{~A} 1, \mathrm{~A} 2, \mathrm{~B}, \mathrm{C} 1$ & UKIRT-Slit2 \\
\hline 01.10 .2006 & UKIRT/CGS4 & 2.1218 & 2700 & 347 & 0.5 & 18500 & jet - knots $1,2,3,4$ & UKIRT-Slit3 \\
\hline 01.10 .2006 & UKIRT/CGS4 & 2.1218 & 2700 & 347 & 0.5 & 18500 & $\mathrm{D}, \mathrm{C} 2$ & UKIRT-Slit4 \\
\hline 06.08 .2006 & TNG/NICS & $0.88-1.45$ & 2000 & 299 & 1 & 500 & $\mathrm{~A}, \mathrm{~B}, \mathrm{C}$ & TNG-Slit1 \\
\hline 06.08 .2006 & TNG/NICS & $1.09-1.80$ & 1800 & 299 & 1 & 500 & $\mathrm{~A}, \mathrm{~B}, \mathrm{C}$ & TNG-Slit1 \\
\hline 06.08 .2006 & TNG/NICS & $1.40-2.47$ & 1800 & 299 & 1 & 500 & $\mathrm{~A}, \mathrm{~B}, \mathrm{C}$ & TNG-Slit1 \\
\hline 06.08 .2006 & TNG/NICS & $1.40-2.47$ & 1800 & 350 & 1 & 500 & jet & TNG-Slit2 \\
\hline 18.10 .2003 & TNG/NICS & $1.09-1.80$ & 2400 & 300 & 1 & 500 & $\mathrm{~A}, \mathrm{~B}, \mathrm{C}$ & TNG-Slit3 \\
\hline 18.10 .2003 & TNG/NICS & $1.40-2.47$ & 2400 & 300 & 1 & 500 & $\mathrm{~A}, \mathrm{~B}, \mathrm{C}$ & TNG-Slit3 \\
\hline 18.10.2003 & TNG/NICS & $1.09-1.80$ & 1800 & 353.5 & 1 & 500 & jet & TNG-Slit4 \\
\hline 18.10 .2003 & TNG/NICS & $1.40-2.47$ & 2400 & 353.5 & 1 & 500 & jet & TNG-Slit4 \\
\hline 11.05.1996 & ISO/SWS & $2.38-45.2$ & 1912 & $\cdots$ & $33 \times 20$ & $1000-2000$ & $\mathrm{~A}, \mathrm{~B}, \mathrm{C}, \mathrm{D}$ & \\
\hline 30.12 .1995 & ISO/LWS & 43-197 & 750 & $\cdots$ & 80 & 200 & A, B, C, D & \\
\hline
\end{tabular}

used grisms overlap between $\sim 1.1$ and $1.8 \mu \mathrm{m}$ (see Table 1), the single spectra were combined to obtain deeper spectra. Finally, since no substantial difference was found between the pairs of spectra from Slits 1 and 3, or from Slits 2 and 4, they were combined together to get a deeper spectrum for each observed knot.

\subsubsection{High-resolution spectroscopy}

Our $\mathrm{H}_{2} 1-0 \mathrm{~S}(1)\left(\lambda_{\mathrm{vac}}=2.1218356 \mu \mathrm{m}\right.$; Bragg et al. 1982) echelle spectra were obtained at UKIRT using the spectrometer CGS4 (Mountain et al. 1990) (see Table 1), equipped with a $256 \times 256$ pixel $\mathrm{InSb}$ array $\left(0 .{ }^{\prime} 41 \times 0.90\right.$ pixel scale, in the dispersion and spatial direction, respectively). A 1-pixel-wide slit was used, resulting in a velocity resolution of $\sim 7.4 \mathrm{~km} \mathrm{~s}^{-1}$ pixel $^{-1}$ and corresponding to a nominal resolving power of $\sim 18500$. The instrumental profile in the dispersion direction, measured from Gaussian fits to the $\mathrm{OH}$ sky lines, was $\sim 10.9 \mathrm{~km} \mathrm{~s}^{-1}$ (or $1.46 \AA$ ).

The targets and position angles (PAs) of the four slits are reported in Table 1, while the slit positions are shown in Fig. 1 and encompass all the $\mathrm{H}_{2}$ knots of the flow. Slits 1 and 2 are parallel to the flow axis and positioned on the knots close to the source (knots A, B, and C). Slit 3 encompasses the so-called "jet", and Slit 4 encompasses knot D and partially knot C. The total exposure time for each position is $2700 \mathrm{~s}$.

The raw spectra were wavelength-calibrated using the bright $\mathrm{OH}$ lines (Rousselot et al. 2000) detected on each frame. The employed IRAF tasks (identify, re-identify, fitcoords, and transform) also correct for the spatial distortion, and a 3rd-order fit in two dimensions has been used. As a result, the calibration is accurate to $\sim 3 \mathrm{~km} \mathrm{~s}^{-1}$. The spectra were not flux-calibrated.

\subsubsection{ISO data archive}

Mid-IR and far-IR spectra of the flow were retrieved from the ISO satellite Data Archive, in the form of Highly Processed Data Product (HPDP set 35500738, by Frieswijk et al.; HPDP set 04300333 by Lloyd, Lerate, and Grundy). The first set of data (from 2.38 to $45.2 \mu \mathrm{m}$, see also Table 1) was taken with the Short Wavelength Spectrometer (SWS, de Graauw et al. 1996) in the AOT01 grating mode ( $\mathcal{R} \sim 1000-2000)$, and is centred on the IRAS source covering a region of $33^{\prime \prime} \times 20^{\prime \prime}$. The covered FoV is shown in Fig. 1. The second set of data (from 43 to $197 \mu \mathrm{m}$ ) was taken with the Long Wavelength Spectrometer (LWS, Clegg et al. 1996) in the AOT01 grating mode $(\lambda / \Delta \lambda \sim 200)$, and is positioned $\sim 48^{\prime \prime}$ north of the source. With a beam of $80^{\prime \prime}$ it covers large parts of the flow, except the so-called "jet".

\section{Results}

\section{1. $\mathrm{H}_{2}$ and [Fe II] imaging}

An overview of the large-scale morphology of the $\mathrm{H}_{2}$ flow is given in Fig. 1. The emission in the SE, the so-called "jet", appears to be composed of four different knots, labelled knots 1 to 4 (see Sect. 3.3). No other knots were detected SE or NW up to $\sim 2$ !.8 (or $\sim 1.4 \mathrm{pc}$ ) from the source, down to a $3 \sigma$ limit of $\sim 8 \times 10^{-17} \mathrm{erg} \mathrm{s}^{-1} \mathrm{~cm}^{-2} \operatorname{arcsec}^{-2}$.

A new emission feature, labelled $\mathrm{X}(\alpha(\mathrm{J} 2000)=$ $20^{\mathrm{h}} 14^{\mathrm{m}} 26.1^{\mathrm{s}}$ and $\left.\delta(\mathrm{J} 2000)=41^{\circ} 13^{\prime} 32^{\prime \prime} \cdot 4\right)$, has been detected close to the position of the IRAS source. Figure 2 shows a close-up in $\mathrm{H}_{2}$ of the source position and the new knot, located in the redshifted lobe. The circle represents the formal error for the NICS plate solution centred on the IRAS source position (Hofner et al. 2007). Contours of the [Fe II] emission are superimposed on this image as well. The two stars in the field have been used to match the $\mathrm{H}_{2}$ and [Fe II] images ex- 


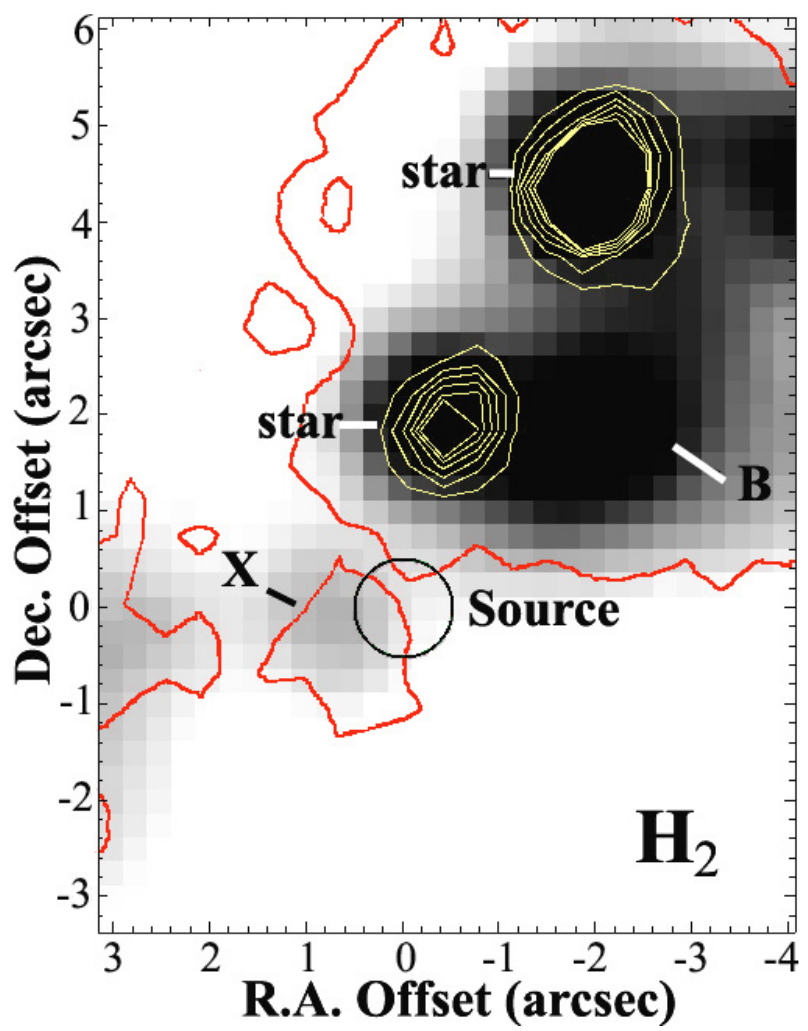

Fig. 2. Close-up of the $\mathrm{H}_{2}$ image around the presumed source position (Hofner et al. 2007, see also the caption of Fig. 1) indicated by a circle (the radius represents the formal error for the NICS plate solution). [Fe II] contours (5 in red, 30 in yellow, 50, 60, 70, 80, 100 $\times \sigma \sim 2.5 \times 10^{-17} \mathrm{erg} \mathrm{s}^{-1} \mathrm{~cm}^{-2} \operatorname{arcsec}^{-2}$ ) are overlaid on the image. The positions of the new knot $\mathrm{X}$, knot $\mathrm{B}$, the source, and two field stars are also indicated.

actly. Both our $\mathrm{H}_{2}-K^{\prime}$ and [Fe II]-H data (as well as the spectroscopic data, see Sect 3.2) indicate that the emission of this knot arises from a shock. Remarkably, the knot overlaps with one of the bipolar features (located SE of the source) detected in the $K$-band by Sridharan et al. (2005). No other [Fe II] emission has been detected along this flow, down to a $3 \sigma$ limit of $\sim 3 \times 10^{-16} \mathrm{erg} \mathrm{s}^{-1} \mathrm{~cm}^{-2} \operatorname{arcsec}^{-2}$ or along the blue lobe at the position where an $\mathrm{H} \alpha$ and [S II] emitting knot was observed by Shepherd et al. (2000).

The higher angular resolution of Subaru images delineates details of the morphology of the $\mathrm{H}_{2}$ flow close to the source. In Fig. 3 we show the continuum-subtracted $\mathrm{H}_{2}$ image. The $\mathrm{Br} \gamma$ image turned out to be more efficient than the $\mathrm{K}_{\text {cont }}$ image in removing the strong continuum detected on the knots. Its use is justified by the absence of any $\operatorname{Br} \gamma$ emission in the observed knots (see Sect. 3.2). Scattered $\mathrm{H}_{2}$ emission would, of course, not be subtracted by this procedure. The $\mathrm{H}_{2}$ flow wiggles noticeably with an opening angle of $\sim 16^{\circ}$ around an axis with PA of $\sim-61^{\circ}$. This fully agrees with $\mathrm{SiO}$ radial velocity observations (Cesaroni et al. 1999; Su et al. 2007), which revealed a precessing jet with an opening cone of $21^{\circ}, \mathrm{PA} \sim-60^{\circ}$, and an inclination of $9^{\circ}$ with respect to the plane of the sky. This small-amplitude precession seems to be superimposed on the larger one observed on a large scale (Shepherd et al. 2000; Cesaroni et al. 2005; see also Fig. 1). In the blue lobe (see Fig. 3) knot B appears composed of a faint jet (feature 1) ending in a bow shock (2), which is preceeded by two smaller bow shocks (3). Further ahead, knot $\mathrm{C}$ consists of at least two bright structures. In the red lobe, the new knot $\mathrm{X}$ is

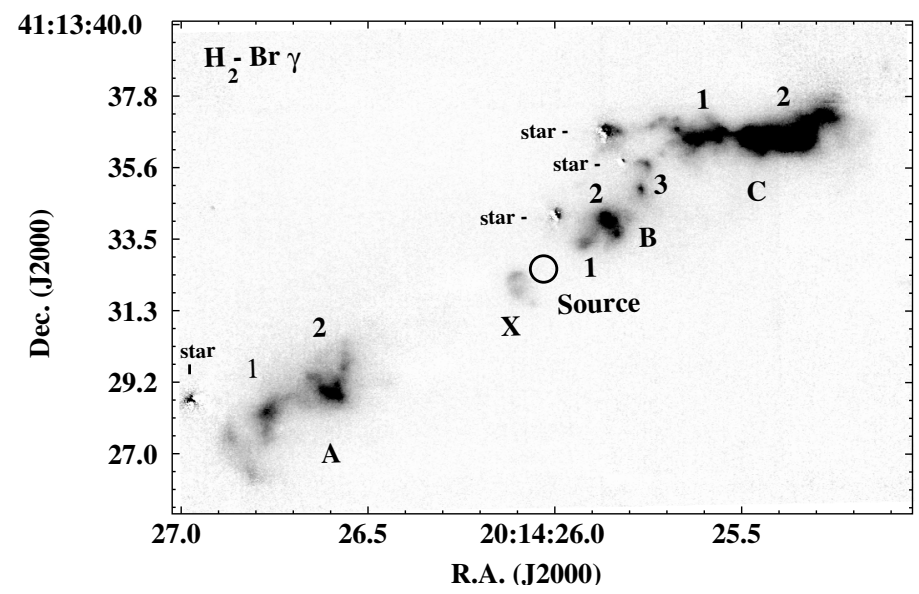

Fig. 3. Continuum-subtracted $\mathrm{H}_{2}-\mathrm{Br} \gamma$ image from Subaru. The $\mathrm{Br} \gamma$ image was used as a continuum, since no $\mathrm{Br} \gamma$ emission is detected on our spectra (see text). The identified knots and the presumed source position (see also the captions of Figs. 1 and 2) are indicated. On this scale, the wiggling structure of the flow becomes visible.

visible as well, and, further out, knot A appears fragmented in 2-3 sub-structures.

\subsection{Low-resolution spectroscopy}

\subsubsection{Low-resolution - TNG}

From our four TNG slits, we derived low-resolution spectra of six knots, namely A, B, C, and X close to the source, and knots 1 and 4 along the "jet". All these spectra are rich in $\mathrm{H}_{2}$ emission. Remarkably, no ionic emission is detected along the flow, with the exception of faint [Fe II] emission in knot X (also observed in the narrow-band image) and in knot B (below the detection limit of our imaging). It is worth noting that the ratio between the $\mathrm{H}_{2}$ lines (see, e.g., $2.12 \mu \mathrm{m}$ ) and the [Fe II] lines changes in the observed knots. Continuum emission from the regions enclosing knots $\mathrm{A}$ and $\mathrm{B}$ is observed in the $K$ band.

The lines detected in the spectra, along with their vacuum wavelengths and fluxes (uncorrected for the extinction), are presented in Table 2. Line fluxes were obtained by fitting the profile with a single or double Gaussian in case of blending. The uncertainties associated with these data derive only from the rms of the local baseline multiplied by the linewidths. Lines showing fluxes with $S / N$ ratio between 2 and 3 have been labelled in the table.

Figure 4 shows the spectrum of knot $\mathrm{C}$, the brightest one observed. Only $\mathrm{H}_{2}$ lines are visible from 1 to $2.5 \mu \mathrm{m}$, involving vibrational levels from $v=1$ up to $v=5$ (coming from energy levels from $6000 \mathrm{~K}$ up to $30000 \mathrm{~K}$ ). The presence of lines from high- $v$ levels (i.e. with high excitation energies) indicates a high excitation of the gas. In the remaining knots, we identify $\mathrm{H}_{2}$ emission lines up to $v \leq 3$, mostly detected in the $H$ - and $K$-bands (except for a few $v=2$ lines from the $J$-band observed in knot B).

Considering both the combined effects of the knot morphologies and the slit positions, there is excellent agreement between the photometric fluxes in our images and the fluxes measured in our spectra for both [Fe II] $(1.64 \mu \mathrm{m})$ and $\mathrm{H}_{2}(2.12 \mu \mathrm{m})$ lines. 


\subsubsection{Low resolution - ISO}

Table 3 lists the lines identified in the ISO-SWS and ISO-LWS spectra, their vacuum wavelengths and integrated fluxes, estimated from Gaussian fits to the (unresolved) line profiles, together with the error from the rms noise of the local baseline. Due to the large FoV of the two instruments, it is impossible to determine the spatial extension of the emitting region, thus the measured fluxes can originate from any or all of the different knots (A...D) close to the source. Several emssion lines are detected, superimposed on a strong continuum observed in both SWS and LWS spectra. The detected lines are shown in Fig. 5. The $\mathrm{H}_{2}$ lines from the $v=1$ and $v=0$ levels have been detected between 2.4 and $17 \mu \mathrm{m}$. In particular, the $1-0 \mathrm{Q}$ lines (also observed in our NIR spectra), the 1-0 O lines, and the $0-0 \mathrm{~S}$ lines, which usually trace the coldest component of the $\mathrm{H}_{2}$ flow (see e.g., Froebrich et al. 2002; Giannini et al. 2004, 2006) were detected. In addition, two bright ionic lines were observed in the LWS spectrum, namely [OI] at $63 \mu \mathrm{m}$ and [C II] at $158 \mu \mathrm{m}$. The origin of these lines is indeed intriguing, because no strong ionic emission lines were detected in the NIR. Possibly, they could originate from the same spot where [Fe II] is detected, and/or in a deeply embedded region (close to the source) not visible at NIR wavelengths, because our spectra do not show any evidence of the bright [C I] doublet at $1 \mu \mathrm{m}$, often detected in low-mass jets (see e.g. Nisini et al. 2002; Giannini et al. 2004). However, a more convincing explanation is that these lines are not arising from the shocks, but from a photo-dissociation region (PDR) around the massive object. Indeed, from the $[\mathrm{OI}](63 \mu \mathrm{m}) /[\mathrm{C} \mathrm{II}](158 \mu \mathrm{m})$ ratio, we can distinguish between the two scenarios (see e.g., Hollenbach $\&$ McKee 1989). For intensity ratios below 10, the PDR origin is favoured. In our LWS spectrum this ratio is close to 1.

\subsection{High resolution spectroscopy}

In Table 4 we report the results of the high-resolution $\mathrm{H}_{2}$ spectroscopy. For each knot in each slit we indicate the $\mathrm{H}_{2}$ radial velocities $\left(v_{\text {rad }}\right)$, corrected for the cloud speed with respect to the LSR ( $v_{\mathrm{LSR}}=-3.5 \mathrm{~km} \mathrm{~s}^{-1}$, Cesaroni et al. 1997). Two or more velocity components are given where detected. The first reported value is the peak value. The last column gives the full width at zero intensity ( $F W Z I$ ) of the line profile, measured where the flux reaches a $2 \sigma$ background noise level (see e.g. Davis et al. 2001).

Position-velocity $(P-V)$ diagrams are presented in Fig. 6 (CGS4 Slits 1 and 2) and in Fig. 7 (CGS4 Slits 3 and 4). In Fig. 8 the emission line profiles are shown. It is worth noting that our spectra often cannot resolve the single structures inside the wiggling jet, due to the limited spatial resolution. As a result, radial velocities are often an average over more than one substructure visible in the Subaru image.

Almost all the knots also have more than one velocity component in their spectral line profile, depending on the position of the slit with respect to the knot. However, such components are never completely resolved (with the exception of knot 2 along the "jet", see Fig. 8), but they instead show up like "bumps" along the smooth profile. When single-peaked profiles are observed, the lines are never symmetric, but evidence of extended line-wing emission (opposite to the blue- or redshifted peak) is always detected. This may be indicative of a bow shock morphology of the knots, producing both the wings and the different velocity components (see e.g. Davis et al. 2001; Schultz et al. 2005), as also indicated by the sub-millimetric observations of Su et al. (2007). Remarkably, knot B has four velocity components (see Table 4 and Fig. 8). The fourth component located at $\sim 64 \mathrm{~km} \mathrm{~s}^{-1}$, and visible along the profile as a "bump" on the redshifted wing (see also knot X profile) cannot be explained in a bow shock context. This component could originate from a different flow, but we have no further evidence in support of this hypothesis.

The flux peaks of the knots range from -42 to $-14 \mathrm{~km} \mathrm{~s}^{-1}$ in the blue lobe, and from -8 to $47 \mathrm{~km} \mathrm{~s}^{-1}$ in the red lobe (see also Table 4). Noticeably, knots X and A in the red lobe (Fig. 6, central panel) have a slightly negative peak velocity. This can be understood by considering that close to the source the axis of the flow has an inclination of $\sim 9^{\circ}$ with respect to the plane of the sky, and the aperture angle of the precessing jet is $\sim 37^{\circ}$. Consequently, even if the knots are located in the "red" lobe, they could have a negative $v_{\text {rad }}$ (see also Su et al. 2007).

In both lobes, the absolute peak radial velocities of knots close to the source (A...C) are lower $\left(0-30 \mathrm{~km} \mathrm{~s}^{-1}\right)$ than those located at greater distances (knot D and knots $1 . .4,40-50 \mathrm{~km} \mathrm{~s}^{-1}$ ). On the other hand, the FWZI of the line profiles decreases with distance (see Table 4 and Fig. 8). The line profiles of the knots close to the source are broader on average $\left(110-180 \mathrm{~km} \mathrm{~s}^{-1}\right)$ than those far from the source $\left(70-90 \mathrm{~km} \mathrm{~s}^{-1}\right)$. This could confirm that the inclination of the flow axis (with respect to the plane of the sky) is different in the two regions; i.e., it changes from $\sim 9^{\circ}$, close to the source, to $\sim 45^{\circ}$ further out, at knots $1 \ldots 4$ and knot D. On a smaller scale, radial velocities appear to oscillate. This is very visible in $P-V$ diagrams of Slit 3, and marginally, for the redshifted knots in Slit 1.

Assuming two different values for the inclination, the spatial velocity of the knots ranges between 50 and $80 \mathrm{~km} \mathrm{~s}^{-1}$.

\subsection{Physical parameters of the gas}

The wealth of molecular hydrogen lines detected in both MIR and NIR spectra allows us to perform a detailed study of the $\mathrm{H}_{2}$ excitation in a high-mass jet, also taking the pure rotational lines into account, for the first time.

As a result, employing all the available $\mathrm{H}_{2}$ ratios in our rovibrational diagrams, we have derived column densities, extinction, and temperature of the gas. Combining these parameters with the $2.12 \mu \mathrm{m}$ flux obtained from the narrow-band imaging, we determined an accurate measurement of the $\mathrm{H}_{2}$ luminosity $\left(L_{\mathrm{H}_{2}}\right)$ for each knot and for the entire flow (see Caratti o Garatti et al. 2006 for a detailed description of this procedure).

\subsubsection{Ro-vibrational diagrams from NIR lines}

As a first step in our analysis, we only employed the NIR lines. Line pairs originating from the same energy level should lie in the same position of the ro-vibrational diagram. By varying the extinction value $\left(A_{\mathrm{V}}\right)$ and increasing the goodness of the fit (maximising the correlation coefficient), extinction and temperature can be evaluated simultaneously (see e.g. Giannini et al. 2004; Davis et al. 2004; Caratti o Garatti et al. 2006). To reduce the uncertainties, only the transitions with $S / N>3$ and not affected by blending with other lines were used. The result of this analysis is shown in Table 5, where the average excitation temperature, the $A_{v}$, and the column densities of the warm gas component are listed for each knot (Cols. 2-4). The ro-vibrational diagrams are shown in Fig. 9.

To compute the extinction in each knot, we selected all the available pairs of lines. From the spectrum of knotC 41 lines were used, giving 29 different ratios (i.e. 
Table 2. Observed lines in the IRAS 20126+4104 outflow: knots A, B, C, X, jet-1, jet-4.

\begin{tabular}{|c|c|c|c|c|c|c|c|}
\hline \multirow{2}{*}{$\begin{array}{c}\text { Term } \\
\mathrm{H}_{2} \text { lines }\end{array}$} & \multirow[t]{2}{*}{$\lambda(\mu \mathrm{m})$} & \multicolumn{6}{|c|}{$F \pm \Delta F\left(10^{-15} \mathrm{erg} \mathrm{cm}^{-2} \mathrm{~s}^{-1}\right)$} \\
\hline & & A & B & $\mathrm{C}$ & $\mathrm{X}$ & knot 1 -jet & knot 4-jet \\
\hline $2-0 \mathrm{~S}(9)$ & 1.053 & $\cdots$ & $\cdots$ & $1.3 \pm 0.4$ & $\cdots$ & $\cdots$ & $\cdots$ \\
\hline $2-0 \mathrm{~S}(8)$ & 1.057 & $\cdots$ & .. & $0.9 \pm 0.3$ & $\cdots$ & .. & $\ldots$ \\
\hline $2-0 \mathrm{~S}(7)$ & 1.064 & $\cdots$ & $\cdots$ & $2.8 \pm 0.4$ & $\cdots$ & $\cdots$ & $\ldots$ \\
\hline $2-0 \mathrm{~S}(6)$ & 1.073 & $\ldots$ & $\ldots$ & $1.6 \pm 0.4$ & $\ldots$ & $\ldots$ & $\ldots$ \\
\hline $2-0 \mathrm{~S}(5)$ & 1.085 & $\ldots$ & ... & $3.5 \pm 0.4$ & $\ldots$ & $\ldots$ & $\ldots$ \\
\hline $2-0 \mathrm{~S}(4)$ & 1.100 & $\ldots$ & ... & $1.8 \pm 0.3$ & $\ldots$ & $\ldots$ & $\ldots$ \\
\hline $2-0 \mathrm{~S}(3)$ & 1.117 & $\ldots$ & $1.3 \pm 0.4$ & $4.3 \pm 0.2$ & .. & $\ldots$ & $\ldots$ \\
\hline $3-1 \mathrm{~S}(9)+3-1 \mathrm{~S}(10)$ & 1.120 & .. & .. & $2.0 \pm 0.2$ & $\ldots$ & .. & $\ldots$ \\
\hline $3-1 \mathrm{~S}(11)$ & 1.122 & $\cdots$ & $\cdots$ & $1.0 \pm 0.3$ & .. & $\cdots$ & $\ldots$ \\
\hline $3-1 \mathrm{~S}(8)$ & 1.125 & $\ldots$ & $\ldots$ & $0.7 \pm 0.2$ & $\ldots$ & $\ldots$ & $\ldots$ \\
\hline $3-1 S(7)$ & 1.130 & $\ldots$ & $\cdots$ & $1.4 \pm 0.2$ & $\cdots$ & ... & $\ldots$ \\
\hline $3-1 S(13)$ & 1.132 & $\ldots$ & ... & $0.5 \pm 0.2:$ & $\ldots$ & $\ldots$ & $\ldots$ \\
\hline $2-0 \mathrm{~S}(2)$ & 1.138 & $\ldots$ & ... & $2.0 \pm 0.2$ & $\ldots$ & $\ldots$ & $\ldots$ \\
\hline $3-1 S(6)$ & 1.140 & $\ldots$ & .. & $0.7 \pm 0.2$ & .. & .. & $\ldots$ \\
\hline $3-1 \mathrm{~S}(5)$ & 1.152 & $\ldots$ & $\ldots$ & $1.9 \pm 0.3$ & $\cdots$ & $\cdots$ & $\cdots$ \\
\hline $2-0 \mathrm{~S}(1)$ & 1.162 & $\cdots$ & $1.3 \pm 0.4$ & $3.5 \pm 0.3$ & $\cdots$ & $\cdots$ & $\ldots$ \\
\hline $3-1 \mathrm{~S}(4)$ & 1.167 & $\ldots$ & $\ldots$ & $1.2 \pm 0.3$ & $\ldots$ & $\cdots$ & $\ldots$ \\
\hline $3-1 \mathrm{~S}(3)$ & 1.186 & $\ldots$ & $\ldots$ & $2.5 \pm 0.3$ & $\ldots$ & $\ldots$ & $\ldots$ \\
\hline $2-0 \mathrm{~S}(0)+4-2 \mathrm{~S}(10)$ & $1.189-1.190$ & $\ldots$ & $\ldots$ & $0.9 \pm 0.2$ & $\ldots$ & $\ldots$ & $\ldots$ \\
\hline $4-2 S(9)+4-2 S(8)$ & $1.196-1.199$ & $\cdots$ & ... & $0.7 \pm 0.2$ & $\ldots$ & $\ldots$ & $\ldots$ \\
\hline 4-2 S(7) & 1.205 & $\cdots$ & $\cdots$ & $1.1 \pm 0.2$ & $\ldots$ & $\cdots$ & $\ldots$ \\
\hline $3-1 \mathrm{~S}(2)$ & 1.207 & $\cdots$ & $\cdots$ & $0.9 \pm 0.2$ & $\cdots$ & $\cdots$ & $\ldots$ \\
\hline $4-2 S(6)$ & 1.214 & $\ldots$ & $\cdots$ & $0.6 \pm 0.2$ & $\cdots$ & $\cdots$ & $\cdots$ \\
\hline $4-2 S(5)$ & 1.226 & $\ldots$ & $\cdots$ & $1.4 \pm 0.2$ & $\cdots$ & $\ldots$ & $\ldots$ \\
\hline $3-1 \mathrm{~S}(1)$ & 1.233 & $\ldots$ & $\ldots$ & $1.4 \pm 0.2$ & $\cdots$ & $\cdots$ & $\ldots$ \\
\hline $2-0 \mathrm{Q}(1)$ & 1.238 & $\ldots$ & $0.6 \pm 0.3:$ & $2.6 \pm 0.2$ & $\ldots$ & $\cdots$ & $\ldots$ \\
\hline $2-0 \mathrm{Q}(2)+4-2 \mathrm{~S}(4)$ & $1.242-1.242$ & $\ldots$ & $\ldots$ & $1.4 \pm 0.2$ & $\ldots$ & $\ldots$ & $\ldots$ \\
\hline $2-0 \mathrm{Q}(3)$ & 1.247 & $\ldots$ & $0.9 \pm 0.4:$ & $2.8 \pm 0.2$ & $\ldots$ & $\ldots$ & $\ldots$ \\
\hline $2-0 \mathrm{Q}(4)$ & 1.254 & $\cdots$ & $\ldots$ & $1.1 \pm 0.2$ & $\cdots$ & $\cdots$ & $\ldots$ \\
\hline $4-2 S(3)+3-1 S(0)$ & 1.261 & $\cdots$ & $\cdots$ & $2.7 \pm 0.3$ & $\cdots$ & $\cdots$ & $\ldots$ \\
\hline $2-0 \mathrm{Q}(5)$ & 1.263 & $\cdots$ & $\cdots$ & $2.1 \pm 0.3$ & $\cdots$ & $\cdots$ & $\ldots$ \\
\hline $2-0 \mathrm{Q}(6)$ & 1.274 & $\cdots$ & $\cdots$ & $0.7 \pm 0.2$ & $\cdots$ & $\cdots$ & $\ldots$ \\
\hline $4-2 S(2)$ & 1.284 & $\ldots$ & $\ldots$ & $0.6 \pm 0.2$ & $\ldots$ & $\ldots$ & $\ldots$ \\
\hline $2-0 \mathrm{Q}(7)$ & 1.287 & $\ldots$ & .. & $1.9 \pm 0.2$ & $\ldots$ & $\ldots$ & $\ldots$ \\
\hline $2-0 \mathrm{Q}(8)$ & 1.302 & $\cdots$ & $\cdots$ & $0.7 \pm 0.2$ & $\cdots$ & $\cdots$ & $\ldots$ \\
\hline $4-2 \mathrm{~S}(1)$ & 1.311 & $\cdots$ & $\cdots$ & $0.9 \pm 0.2$ & $\ldots$ & $\cdots$ & $\ldots$ \\
\hline $5-3 \mathrm{~S}(5)$ & 1.312 & $\cdots$ & $\cdots$ & $0.7 \pm 0.2$ & $\cdots$ & $\cdots$ & $\ldots$ \\
\hline $3-1 \mathrm{Q}(1)$ & 1.314 & .. & $\cdots$ & $1.2 \pm 0.2$ & $\ldots$ & $\ldots$ & $\ldots$ \\
\hline $3-1 \mathrm{Q}(2)+2-0 \mathrm{Q}(9)$ & $1.318-1.319$ & $\cdots$ & $\cdots$ & $1.6 \pm 0.2$ & $\cdots$ & $\cdots$ & $\ldots$ \\
\hline $3-1 \mathrm{Q}(3)$ & 1.324 & $\cdots$ & $\cdots$ & $1.3 \pm 0.2$ & $\ldots$ & $\ldots$ & $\ldots$ \\
\hline $3-1 \mathrm{Q}(4)$ & 1.333 & $\ldots$ & $\ldots$ & $0.5 \pm 0.2:$ & $\ldots$ & $\ldots$ & $\ldots$ \\
\hline $2-0 \mathrm{O}(3)$ & 1.335 & $\ldots$ & $\ldots$ & $2.7 \pm 0.2$ & $\ldots$ & $\ldots$ & $\ldots$ \\
\hline $3-1 \mathrm{Q}(5)+4-2 \mathrm{~S}(0)$ & $1.342-1.342$ & $\cdots$ & $\cdots$ & $1.6 \pm 0.2$ & $\cdots$ & $\cdots$ & $\ldots$ \\
\hline 5-3 $S(3)$ & 1.347 & $\cdots$ & $\cdots$ & $0.7 \pm 0.2$ & $\cdots$ & $\cdots$ & $\cdots$ \\
\hline $3-1 \mathrm{O}(3)+4-2 \mathrm{Q}(4)$ & 1.418 & $\ldots$ & ... & $1.3 \pm 0.4$ & $\ldots$ & $\ldots$ & $\ldots$ \\
\hline $4-1 \mathrm{Q}(5)+2-0 \mathrm{O}(5)$ & $1.430-1.432$ & $\cdots$ & $\cdots$ & $2.1 \pm 0.4$ & $\cdots$ & $\cdots$ & $\ldots$ \\
\hline $2-0 \mathrm{O}(6)$ & 1.487 & $\ldots$ & $\ldots$ & $1.0 \pm 0.4:$ & $\ldots$ & $\ldots$ & $\ldots$ \\
\hline 3-1 Q(13) & 1.502 & $\ldots$ & ... & $0.6 \pm 0.3:$ & $\ldots$ & $\ldots$ & $\ldots$ \\
\hline $3-1 \mathrm{O}(5)$ & 1.522 & $\cdots$ & $\cdots$ & $1.3 \pm 0.4$ & $\ldots$ & $\cdots$ & $\ldots$ \\
\hline $2-0 \mathrm{O}(7)$ & 1.545 & $\ldots$ & $\ldots$ & $0.7 \pm 0.3:$ & $\ldots$ & $\ldots$ & $\ldots$ \\
\hline $4-2 \mathrm{O}(5)$ & 1.622 & $\ldots$ & $\ldots$ & $0.9 \pm 0.3$ & $\ldots$ & $\ldots$ & $\ldots$ \\
\hline $3-1 \mathrm{O}(7)$ & 1.645 & $\ldots$ & $\ldots$ & $0.7 \pm 0.2$ & $\ldots$ & $\ldots$ & $\ldots$ \\
\hline $1-0 \mathrm{~S}(11)$ & 1.650 & $\ldots$ & $\ldots$ & $1.0 \pm 0.3$ & $\ldots$ & $\ldots$ & $\ldots$ \\
\hline $1-0 \mathrm{~S}(10)$ & 1.666 & $\ldots$ & $\ldots$ & $1.1 \pm 0.3$ & $\ldots$ & $\ldots$ & $\cdots$ \\
\hline $1-0 \mathrm{~S}(9)$ & 1.688 & $1.9 \pm 0.4$ & $2.1 \pm 0.3$ & $7.4 \pm 0.3$ & $\ldots$ & $0.9 \pm 0.3$ & $0.7 \pm 0.3:$ \\
\hline $1-0 \mathrm{~S}(8)$ & 1.715 & $1.4 \pm 0.4$ & $1.2 \pm 0.2$ & $5.2 \pm 0.3$ & $\ldots$ & $0.7 \pm 0.3:$ & $0.7 \pm 0.3:$ \\
\hline $1-0 \mathrm{~S}(7)$ & 1.748 & $12.1 \pm 0.4$ & $7.5 \pm 0.2$ & $33.7 \pm 0.3$ & $1.9 \pm 0.2$ & $3.8 \pm 0.3$ & $2.5 \pm 0.3$ \\
\hline $1-0 \mathrm{~S}(6)$ & 1.788 & $7.9 \pm 0.4$ & $5.0 \pm 0.2$ & $22.8 \pm 0.3$ & $1.4 \pm 0.2$ & $2.4 \pm 0.3$ & $1.5 \pm 0.4$ \\
\hline 2-1 S(9) & 1.790 & $\ldots$ & $\ldots$ & $1.0 \pm 0.5:$ & $\ldots$ & $\ldots$ & $\ldots$ \\
\hline $2-1 \mathrm{~S}(8)$ & 1.818 & $\ldots$ & $\ldots$ & $1.0 \pm 0.5:$ & $\ldots$ & $\ldots$ & $\ldots$ \\
\hline $1-0 \mathrm{~S}(5)$ & 1.836 & $54 \pm 5$ & $31 \pm 5$ & $125 \pm 5$ & $\ldots$ & $\ldots$ & $\ldots$ \\
\hline $2-1 S(7)$ & 1.853 & $\ldots$ & $\ldots$ & $8.0 \pm 5:$ & $\ldots$ & $\ldots$ & $\ldots$ \\
\hline $1-0 \mathrm{~S}(4)$ & 1.891 & $28 \pm 5$ & $16 \pm 5:$ & $66 \pm 5$ & $\ldots$ & $\ldots$ & $\ldots$ \\
\hline 2-1 S(7) & 1.945 & $\ldots$ & $\ldots$ & & 1.969 & $\ldots$ & $\ldots$ \\
\hline
\end{tabular}


Table 2. continued.

\begin{tabular}{|c|c|c|c|c|c|c|c|}
\hline \multirow{2}{*}{$\begin{array}{c}\text { Term } \\
\mathrm{H}_{2} \text { lines }\end{array}$} & \multirow[t]{2}{*}{$\lambda(\mu \mathrm{m})$} & \multicolumn{6}{|c|}{$F \pm \Delta F\left(10^{-15} \mathrm{erg} \mathrm{cm}^{-2} \mathrm{~s}^{-1}\right)$} \\
\hline & & A & B & $\mathrm{C}$ & $\mathrm{X}$ & knot 1-jet & knot 4-jet \\
\hline $12.0 \pm 5:$ & . & $\cdots$ & & & & & \\
\hline $1-0 \mathrm{~S}(3)$ & 1.958 & $24 \pm 2$ & $51 \pm 5$ & $110 \pm 5$ & $13 \pm 4$ & $\ldots$ & .. \\
\hline $3-2 S(7)$ & $1.7 \pm 0.8$ & $\ldots$ & $\ldots$ & $\ldots$ & & & \\
\hline $2-1 S(4)$ & 2.004 & $\cdots$ & $1.3 \pm 0.4$ & $8.3 \pm 0.8$ & $\cdots$ & $0.6 \pm 0.3:$ & $\cdots$ \\
\hline $3-2 \mathrm{~S}(6)$ & 2.013 & $\ldots$ & $\ldots$ & $1.2 \pm 0.5:$ & $\ldots$ & $\ldots$ & $\ldots$ \\
\hline $1-0 \mathrm{~S}(2)$ & 2.034 & $38.9 \pm 0.5$ & $21.1 \pm 0.4$ & $83.4 \pm 0.5$ & $4.2 \pm 0.4$ & $7.7 \pm 0.3$ & $5.7 \pm 0.4$ \\
\hline $3-2 \mathrm{~S}(5)$ & 2.066 & $0.9 \pm 0.3$ & $0.7 \pm 0.3:$ & $2.9 \pm 0.4$ & $\ldots$ & $\ldots$ & $\ldots$ \\
\hline $2-1 \mathrm{~S}(3)$ & 2.073 & $7.4 \pm 0.4$ & $4.8 \pm 0.3$ & $24.4 \pm 0.4$ & $1.4 \pm 0.4$ & $2.0 \pm 0.4$ & $1.1 \pm 0.3$ \\
\hline $1-0 \mathrm{~S}(1)$ & 2.122 & $121 \pm 0.4$ & $66.4 \pm 0.4$ & $246 \pm 0.4$ & $11.1 \pm 0.4$ & $22.6 \pm 0.4$ & $16.6 \pm 0.4$ \\
\hline $2-1 \mathrm{~S}(2)$ & 2.154 & $3.7 \pm 0.4$ & $2.2 \pm 0.4$ & $8.4 \pm 0.4$ & $0.5 \pm 0.2:$ & $1.0 \pm 0.3$ & $0.6 \pm 0.2$ \\
\hline $3-2 S(3)$ & 2.201 & $1.6 \pm 0.4$ & $0.8 \pm 0.3:$ & $4.3 \pm 0.4$ & $0.4 \pm 0.1:$ & $0.5 \pm 0.2:$ & $\ldots$ \\
\hline $1-0 \mathrm{~S}(0)$ & 2.223 & $30.1 \pm 0.4$ & $15.6 \pm 0.4$ & $59.5 \pm 0.4$ & $2.8 \pm 0.3$ & $5.7 \pm 0.3$ & $4.6 \pm 0.4$ \\
\hline $2-1 S(1)$ & 2.248 & $10.9 \pm 0.4$ & $5.5 \pm 0.4$ & $23.0 \pm 0.4$ & $1.6 \pm 0.4$ & $2.5 \pm 0.3$ & $2.1 \pm 0.4$ \\
\hline $3-2 \mathrm{~S}(2)$ & 2.286 & $0.9 \pm 0.4:$ & $\ldots$ & $1.5 \pm 0.4$ & $\ldots$ & $\ldots$ & $\ldots$ \\
\hline $2-1 \mathrm{~S}(0)$ & 2.355 & $2.7 \pm 0.5$ & $1.7 \pm 0.5$ & $5.1 \pm 0.5$ & $\ldots$ & $\ldots$ & $\ldots$ \\
\hline $3-2 \mathrm{~S}(1)$ & 2.386 & $1.0 \pm 0.5:$ & $\ldots$ & $4.0 \pm 0.5$ & $\ldots$ & $\ldots$ & $\ldots$ \\
\hline $1-0 \mathrm{Q}(1)$ & 2.407 & $135 \pm 5$ & $66 \pm 5$ & $234 \pm 5$ & $11 \pm 3$ & $21 \pm 5$ & $16 \pm 5$ \\
\hline $1-0 \mathrm{Q}(2)$ & 2.413 & $50 \pm 5$ & $25 \pm 5$ & $89 \pm 5$ & $\ldots$ & $\ldots$ & $\ldots$ \\
\hline $1-0 \mathrm{Q}(3)$ & 2.424 & $123 \pm 5$ & $57 \pm 5$ & $218 \pm 5$ & $11 \pm 3$ & $20 \pm 5$ & $13 \pm 5:$ \\
\hline $1-0 \mathrm{Q}(4)$ & 2.437 & $35 \pm 5$ & $18 \pm 5$ & $70 \pm 5$ & $\ldots$ & $\ldots$ & $\ldots$ \\
\hline $1-0 \mathrm{Q}(5)$ & 2.455 & $39 \pm 5$ & $27 \pm 5$ & $142 \pm 5$ & $\cdots$ & $\cdots$ & $\cdots$ \\
\hline \multicolumn{8}{|l|}{$[\mathrm{Fe} \mathrm{II}]$ lines } \\
\hline $\mathrm{a}^{4} \mathrm{D}_{7 / 2}-\mathrm{a}^{6} \mathrm{D}_{9 / 2}$ & 1.257 & $\cdots$ & $0.6 \pm 0.3^{a}$ & $\cdots$ & $1.2 \pm 0.3$ & $\cdots$ & $\cdots$ \\
\hline$a^{4} D_{7 / 2}-a^{6} D_{7 / 2}$ & 1.321 & $\ldots$ & $\ldots$ & $\ldots$ & $0.6 \pm 0.3^{a}$ & $\ldots$ & $\ldots$ \\
\hline$a^{4} D_{7 / 2}-a^{4} F_{9 / 2}$ & 1.644 & $\ldots$ & $1.7 \pm 0.2$ & $\ldots$ & $2.0 \pm 0.2$ & $\ldots$ & $\ldots$ \\
\hline$a^{4} D_{7 / 2}-a^{4} F_{7 / 2}+a^{4} P_{5 / 2}-a^{4} D_{7 / 2}$ & $1.810-1.811$ & $\ldots$ & $1.2 \pm 0.4$ & $\ldots$ & $1.5 \pm 0.4$ & $\ldots$ & $\ldots$ \\
\hline
\end{tabular}

Notes: $3 \sigma$ upper limits (in knot $\mathrm{C}$ ) for the [C I] doublet (at $0.983-5 \mu \mathrm{m}$ ) and for the Br $\gamma$ line are $10^{-15}$ and $6 \times 10^{-16} \mathrm{erg} \mathrm{cm}^{-2} \mathrm{~s}^{-1}$, respectively.

IRAS20126+4104 Knot C

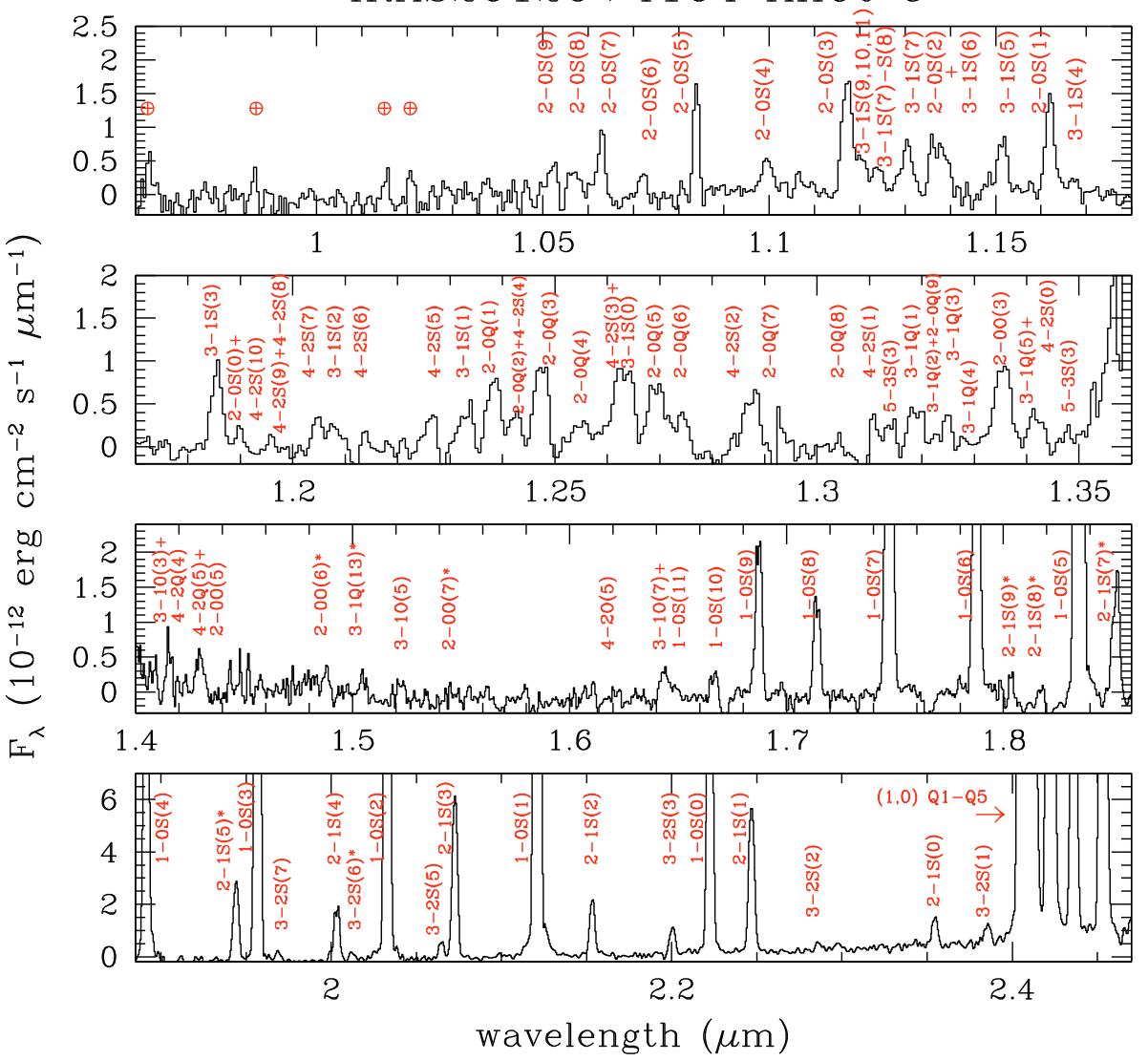

Fig. 4. The 0.9-2.5 $\mu \mathrm{m}$ low-resolution spectrum of knot C in the IRAS $20126+4104$ jet. An asterisk near the line identification marks the detections between 2 and 3 sigma. Telluric lines are indicated by the symbol " $\oplus$ ". 
Table 3. Observed lines in the IRAS 20126+4104 outflow: ISO lines.

\begin{tabular}{ccc}
\hline \hline Term & $\lambda(\mu \mathrm{m})$ & $F \pm \Delta F\left(10^{-13} \mathrm{erg} \mathrm{cm}^{-2} \mathrm{~s}^{-1}\right)$ \\
$\mathrm{H}_{2}$ lines & & Flux \\
\hline $1-0 \mathrm{Q}(1)$ & 2.407 & $1.8 \pm 0.4$ \\
$1-0 \mathrm{Q}(2)$ & 2.413 & $1.4 \pm 0.4$ \\
$1-0 \mathrm{Q}(3)$ & 2.424 & $0.8 \pm 0.4$ \\
$1-0 \mathrm{Q}(4)$ & 2.437 & $0.7 \pm 0.3:$ \\
$1-0 \mathrm{Q}(5)$ & 2.454 & $1.4 \pm 0.3$ \\
$1-0 \mathrm{Q}(6)$ & 2.476 & $1.3 \pm 0.2$ \\
$1-0 \mathrm{Q}(7)$ & 2.500 & $1.4 \pm 0.1$ \\
$1-0 \mathrm{O}(2)$ & 2.627 & $0.6 \pm 0.1$ \\
$1-0 \mathrm{O}(3)$ & 2.803 & $0.7 \pm 0.1$ \\
$1-0 \mathrm{O}(4)$ & 3.004 & $0.6 \pm 0.2$ \\
$1-0 \mathrm{O}(5)$ & 3.235 & $0.8 \pm 0.1$ \\
$0-0 \mathrm{~S}(6)$ & 6.109 & $21 \pm 3$ \\
$0-0 \mathrm{~S}(5)$ & 6.909 & $21 \pm 3$ \\
$0-0 \mathrm{~S}(4)$ & 8.026 & $30 \pm 3$ \\
$0-0 \mathrm{~S}(3)$ & 9.665 & $32 \pm 6$ \\
$0-0 \mathrm{~S}(2)$ & 12.278 & $26 \pm 8$ \\
$0-0 \mathrm{~S}(1)$ & 17.034 & $56 \pm 8$ \\
\hline ionic lines & & \\
{$[\mathrm{O} \mathrm{I}] \mathrm{a}^{3} \mathrm{P}_{1}-\mathrm{a}^{3} \mathrm{P}_{2}$} & 63.18 & $260 \pm 10$ \\
{$[\mathrm{C}$ II $] \mathrm{a}^{3} \mathrm{P}_{3 / 2}-\mathrm{a}^{3} \mathrm{P}_{1 / 2}$} & 157.74 & $370 \pm 10$ \\
\hline
\end{tabular}

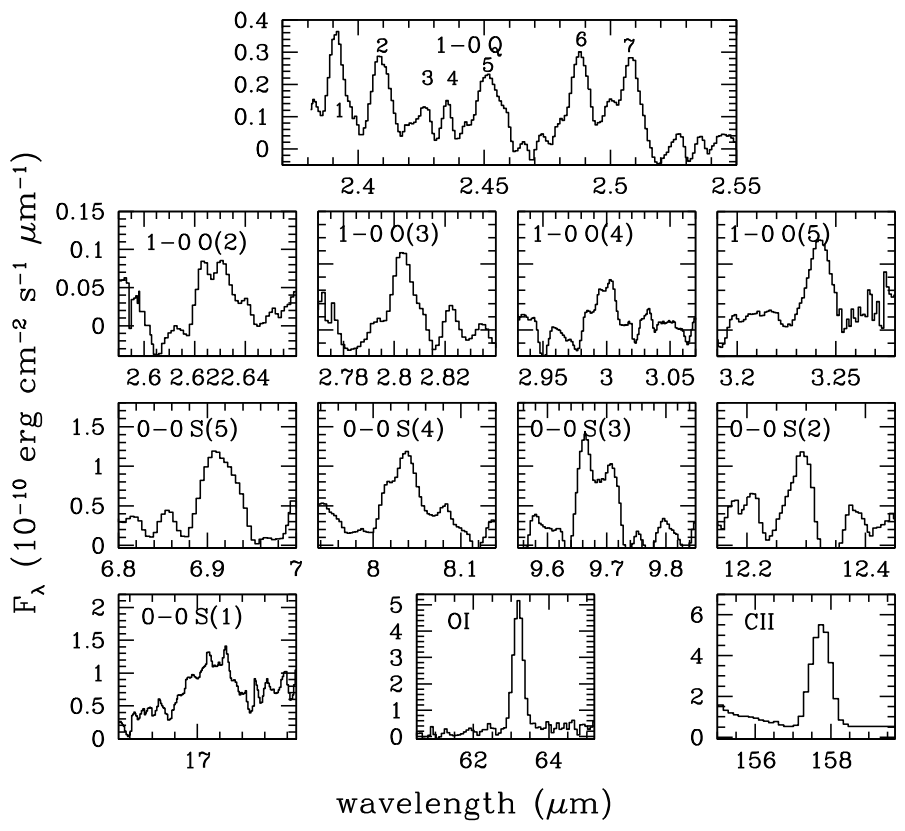

Fig. 5. ISO-SWS and LWS detected lines.

the ratios are among the following groups of transitions: $1-0 \mathrm{~S}(\mathrm{i}), 1-0 \mathrm{Q}(\mathrm{i}+2) ; 2-0 \mathrm{~S}(\mathrm{i}), 2-0 \mathrm{Q}(\mathrm{i}+2), 2-0 \mathrm{O}(\mathrm{i}+4), 2-1 \mathrm{~S}(\mathrm{i}) ; 3-$ $1 \mathrm{~S}(\mathrm{i}), 3-1 \mathrm{Q}(\mathrm{i}+2), 3-1 \mathrm{O}(\mathrm{i}+4)$; with $i=0,1,2 \ldots)$. The best fit was obtained excluding the $1-0 \mathrm{~S}(\mathrm{i}) / 1-0 \mathrm{Q}(\mathrm{i}+2)$ pairs, which deviated more than $3 \sigma$ from the average value. The final value is $A_{\mathrm{v}}=7.6 \pm 0.2$, and it has a very small error. For the other knots, the $A_{\mathrm{v}}$ ranges from 6 to $10 \mathrm{mag}$, with errors between 1 and 3 mag (see Table 5, Col. 3). For these knots, a few pairs of lines were available making the errors larger. With the exception of knot $\mathrm{C}$, all the lines in our ro-vibrational diagrams are well-fitted by a single straight line (see Fig. 9), indicating a uniform temperature of the gas. The observed temperatures range from 2000 to $2500 \mathrm{~K}$.

On the other hand, the different $\mathrm{H}_{2}$ lines detected in knot $\mathrm{C}$ cannot be fitted by a single line. The ro-vibrational diagram exhibits a typical curvature indicating the presence of a
Table 4. $\mathrm{H}_{2}$ radial velocities of individual knots in the IRAS 20126+4104 flow from CGS4 observations.

\begin{tabular}{|c|c|c|}
\hline \multicolumn{3}{|c|}{ CGS4 slit 1} \\
\hline knot & $\begin{array}{c}v_{\text {rad }} \\
\left(\mathrm{km} \mathrm{s}^{-1}\right)\end{array}$ & $\begin{array}{c}\text { FWZI } \\
\left(\mathrm{km} \mathrm{s}^{-1}\right)\end{array}$ \\
\hline A1 & $2 \pm 3$ & 110 \\
\hline A2 & $9 \pm 3$ & 150 \\
\hline$X$ & $-8 \pm 3 ;-28 \pm 3 ; 53 \pm 3$ & 175 \\
\hline B & $-14 \pm 3$ & 160 \\
\hline $\mathrm{C} 2$ & $-25 \pm 3$ & 160 \\
\hline & CGS4 slit 2 & \\
\hline A1 & $-1 \pm 3 ;-52 \pm 3$ & 130 \\
\hline $\mathrm{A} 2$ & $9 \pm 3 ;-12 \pm 3 ; 53 \pm 3$ & 180 \\
\hline $\mathrm{B}$ & $-14 \pm 3 ;-46 \pm 3 ; 26 \pm 3 ; 64 \pm 3$ & 170 \\
\hline $\mathrm{C} 1$ & $-19 \pm 3$ & 130 \\
\hline & CGS4 slit 3 & \\
\hline jet knot 1 & $47 \pm 3 ; 19 \pm 3$ & 70 \\
\hline jet knot 2 & $27 \pm 3$ & 75 \\
\hline jet knot 3 & $41 \pm 3 ; 2 \pm 3$ & 90 \\
\hline jet knot 4 & $6 \pm 3 ; 36 \pm 3$ & 90 \\
\hline & CGS4 slit 4 & \\
\hline $\mathrm{D}$ & $-42 \pm 3 ;-17 \pm 3$ & 70 \\
\hline $\mathrm{C} 2$ & $-28 \pm 3 ;-5 \pm 3 ; 3 \pm 3$ & 90 \\
\hline
\end{tabular}

stratification in the gas temperature. A more elaborate model of a mixture of gas at two different temperatures can describe these data (see e.g. Giannini et al. 2002; Caratti o Garatti et al. 2006; Gredel 2007). The population densities of the lines coming from levels with an excitation energy up to $\sim 12000 \mathrm{~K}$ are consistent with an excitation temperture of $2050 \mathrm{~K}$. These lines indeed trace the warm component of the gas. Conversely, lines with a higher excitation energy $(>12000 \mathrm{~K})$ are thermalised at a higher temperture of $\sim 5200 \mathrm{~K}$. These lines trace the hot component of the gas. A single fit through all the lines only gives a measure of the "averaged" temperature ( $\sim 3300 \mathrm{~K})$ (see Fig. 9, top left panel). In our model the hot component is a fraction of the gas of about $8 \%$.

Another important parameter that derives from rovibrational diagrams is the column density of the gas. The column densities of the warm $\mathrm{H}_{2}$ component are reported in Table 5. They are very similar to those measured in other high-mass protostellar jets $\left(10^{18}-10^{19} \mathrm{~cm}^{-2}\right.$ ) (see e.g. Davis et al. 2004; Gredel 2006), and they are 1-2 orders of magnitude higher than the values observed in low-mass jets.

Finally, from the analysis of Fig. 9, it is worth noting that the $\mathrm{H}_{2}$ gas along the flow is fully thermalised. That we detect no lines with $v \geq 6$ indicates that fluorescence mechanisms do not play an important role in the excitation (see e.g. Black \& van Dishoeck 1987). Excitation by non-thermal processes like fluorescence in the presence of a UV field would cause the vibrational temperature to be different from the rotational temperatures, and thus lines belonging to different vibrational series should not be located on the same line (see e.g. Hora \& Latter 1994). As a consequence, we should observe strong deviations from the smooth (or linear) Boltzmann distribution of our rovibrational diagrams (see also Gredel 2007). Apparently, this contrasts with results of Sect. 3.2.2, where the presence of a PDR was inferred. Such a PDR could produce enough far-UV radiation to excite the higher vibrational levels of the $\mathrm{H}_{2}$ and induce a fluorescent emission (see e.g. Burton 1992), which we do not detect. To evaluate a possible contribution of the PDR 

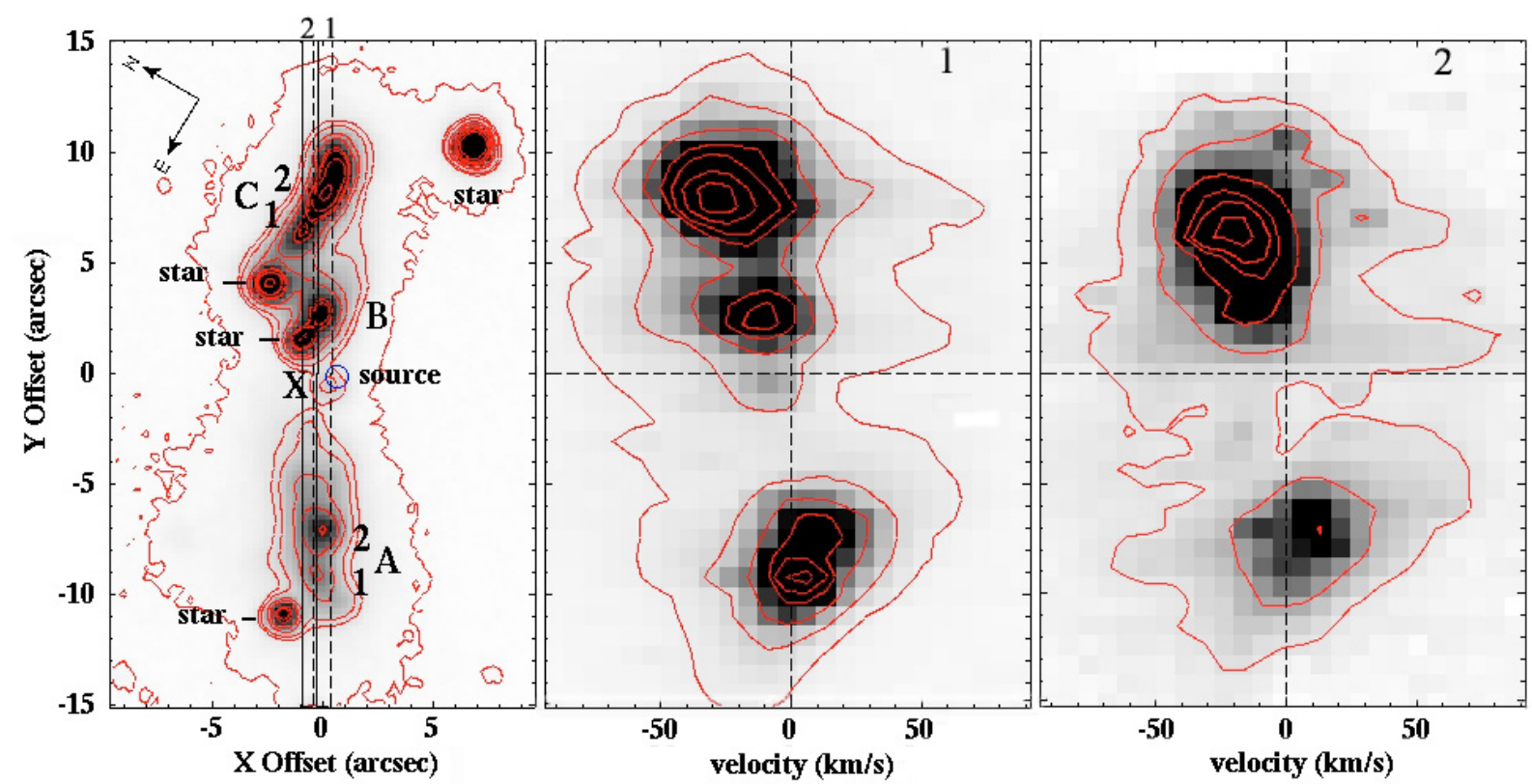

Fig. 6. $P-V$ diagram for slits 1 and 2. The left panel shows the $\mathrm{H}_{2}$ (TNG) image with the slits superimposed, and the position of the knots. $Y$-offsets are displayed from the position of the source. The contour levels are 3, 30, 50, 100, 130, 210, 235, 300, 420× the standard deviation to the mean background $\left(\sigma \sim 2 \times 10^{-17} \mathrm{erg} \mathrm{s}^{-1} \mathrm{~cm}^{-2} \operatorname{arcsec}^{-2}\right)$. The central and right panels display the uncalibrated $\mathrm{H}_{2}(2.12 \mu \mathrm{m}) \mathrm{spectra}$ from CGS4 (slits 1 and 2, respectively). The contour levels are 5, 20, 60, 125, 180, 300, 450× the standard deviation to the mean background.

to the observed lines, we used the "PDR toolbox"3. The observed [C II] $158 \mu \mathrm{m}$ diffuse line intensity (considering an LWS beam-width of $80^{\prime \prime}$ ) is $\sim 2.5 \times 10^{-4} \mathrm{erg} \mathrm{s}^{-1} \mathrm{~cm}^{-2} \mathrm{sr}^{-1}$. Assuming that all the emission arises from the PDR and that the density is $10^{5}-10^{6} \mathrm{~cm}^{-2}$ (Cesaroni et al. 1999), we obtain a relatively faint FUV flux of $G_{0}$ between 10 and 100 (where $G_{0}$ is measured in units of $1.6 \times 10^{-3} \mathrm{erg} \mathrm{s}^{-1} \mathrm{~cm}^{-2}$ ). Under these circumstances the PDR surface temperture does not exceed $100 \mathrm{~K}$ (Kaufman et al. 1999) and the contribution to the overall $\mathrm{H}_{2}$ emission is negligible. For example, the diffuse line intensity of the $0-0 \mathrm{~S}(1)$ is between $10^{-6}$ and $10^{-5} \mathrm{erg} \mathrm{s}^{-1} \mathrm{~cm}^{-2} \mathrm{sr}^{-1}$, while we obtain $\sim 6 \times 10^{-4} \mathrm{erg} \mathrm{s}^{-1} \mathrm{~cm}^{-2} \mathrm{sr}^{-1}$ from our observations (dividing by the ISO-SWS FoV). In the NIR the emission from the shock (at $2.12 \mu \mathrm{m}$ ) is four orders of magnitude greater. These results clearly indicate that the detected $\mathrm{H}_{2}$ arises mostly from shocks.

\subsubsection{The cold $\mathrm{H}_{2}$ component from MIR lines}

As a second step in our analysis, we also used the ISO-SWS lines to study the cold component of the gas, which is usually traced by lines with excitation energy lower than $5000 \mathrm{~K}$, namely the $0-0 \mathrm{~S}$ lines (with $J_{\text {fin }} \leq 5$ ) between 6 and $28 \mu \mathrm{m}$ in the ISO-SWS spectrum.

Since we do not know the spatial extent of the emitting region, we matched the column densities of the $1-0 \mathrm{Q}$ lines present in both NICS and SWS spectra, to intercalibrate the data in the ro-vibrational diagram. Indeed the measured ISO-SWS fluxes of the $1-0 \mathrm{Q}$ lines are very close to those measured in knot $\mathrm{C}$. Therefore we have assumed that its column density is representative of the warm $\mathrm{H}_{2}$ component detected with ISO, as well. The result is shown in Fig. 10. The SWS lines coming from the

3 The "PDR Toolbox" is available at http://dustem. astro. umd. edu and contains downloadable FIR line diagnostic information about PDRs. The tool has been created by Mundy, Wolfire, Lord, and Pound, and it is based on the new PDR models of Kaufman et al. (1999). $v=0$ and $v=1$ levels are overplotted on the ro-vibrational diagram of knot $\mathrm{C}$ (shown in Fig. 9, top left panel). Although there is some scatter among the $v=1$ data points (two $1-0 \mathrm{Q}$ and $1-0 \mathrm{O}$ lines have slightly higher and lower column densities, respectively), the overall fit is quite satisfactory. In particular, the 0-0 S lines reveal the presence of a cold gas component at about $520 \mathrm{~K}$, which has a higher column density than the other components $\left(N_{\mathrm{H}_{2}} \sim 9.7 \times 10^{21} \mathrm{~cm}^{-2}\right.$, see also Fig. 10).

To reproduce the observed ro-vibrational diagram, we calculated a theoretical $\mathrm{H}_{2}$ spectrum for a mixture of three $\mathrm{H}_{2}$ layers in LTE condition at a temperature of 520, 2050, and $5200 \mathrm{~K}$. The adopted LTE code (see also Caratti o Garatti et al. 2006) computes the line intensities involving levels with $0 \leq v \leq 14$ and $0 \leq J \leq 29\left(E_{v, J} \leq 50000 \mathrm{~K}\right)$. Ro-vibrational energies were taken from Dabrowsy (1984) and the Einstein coefficients from Wolniewicz et al. (1998). We assumed an ortho/para ratio equal to three (as also evinced from our ro-vibrational diagrams). The resulting model is plotted in Fig. 10, and properly fits our observations. In this case the warm and hot components are only a small fraction of the total $\mathrm{H}_{2}$ gas, i.e. less than $1 \%$.

\subsection{3. $\mathrm{H}_{2}$ luminosity}

In the last step of our analysis, we inferred $L_{\mathrm{H}_{2}}$. Once the physical parameters $\left(A_{\mathrm{v}}, T\right)$ for each knot were derived, we dereddened the $2.12 \mu \mathrm{m}$ flux obtained from the imaging (see Table 5, Col. 5), adopting the Rieke \& Lebofsky (1985) reddening law, and computed the line ratios with the other $\mathrm{H}_{2}$ lines by applying our radiative LTE code at $T=T_{\text {avg. }}$. From these ratios, the absolute intensities of individual lines were computed and the $\mathrm{H}_{2}$ luminosity of each knot was derived (see Table 5, Col. 6). The sum of these values gives a total $L_{\mathrm{H}_{2}}$ of the flow of $4.6 \pm 0.3 L_{\odot}$. An average temperature reproduces the contribution of both the warm and hot gas components to the radiated energy in $\mathrm{H}_{2}$ well (see Caratti o Garatti et al. 2006). We are not taking the luminosity of the "cold" component into account, which, however, 

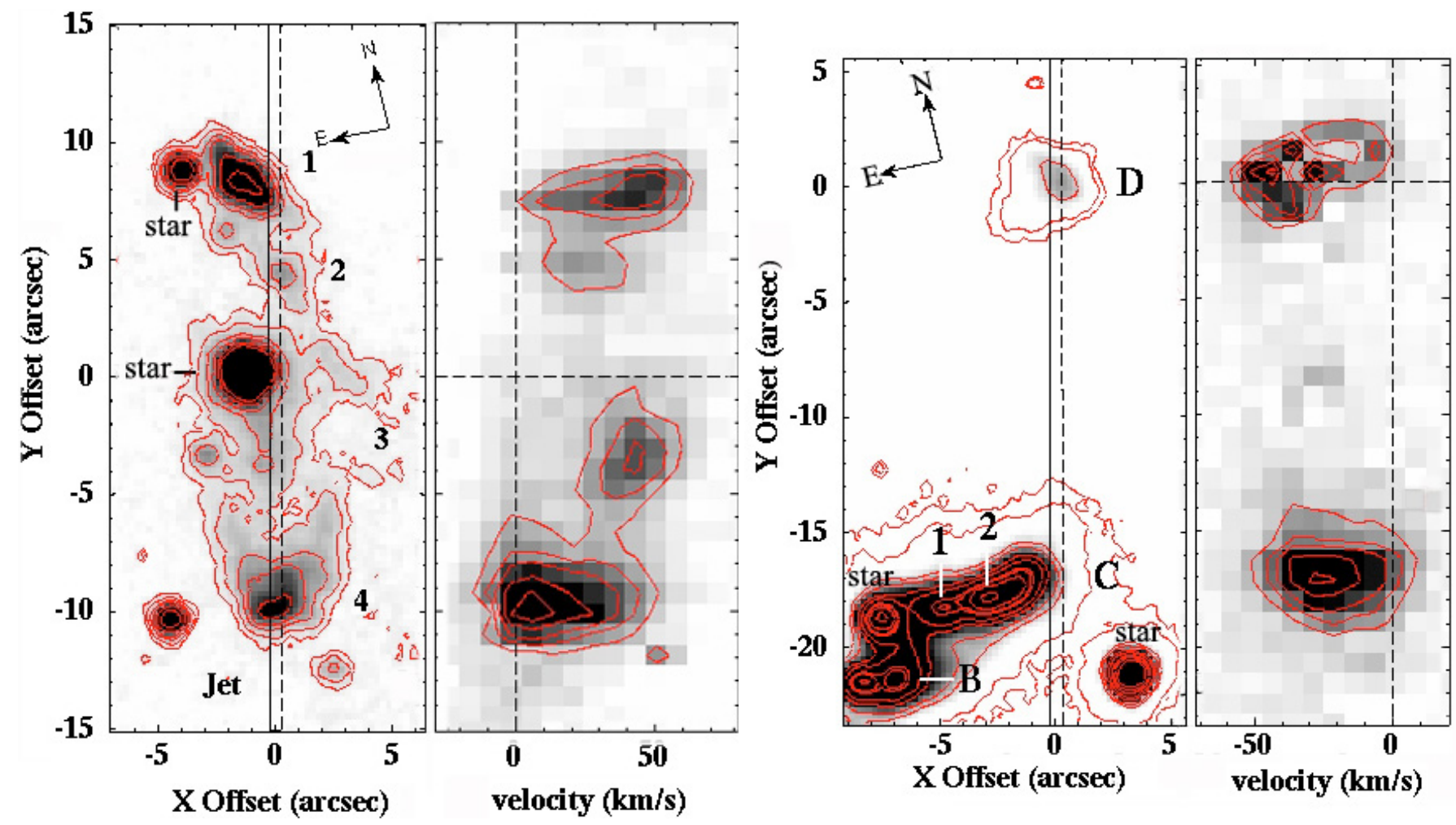

Fig. 7. Left $P-V$ diagram for slit 3. On the left panel the $\mathrm{H}_{2}$ (TNG) image indicates the position of the slit and knots. $Y$ offsets are displayed from the position of the star in the centre of the image. The contour levels are $3,6,12,20,30,50 \times \sigma$. On the right, the uncalibrated $\mathrm{H}_{2}(2.12 \mu \mathrm{m})$

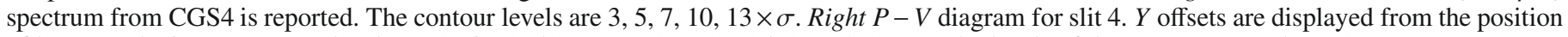

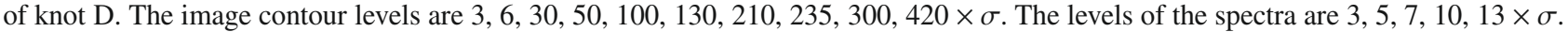

here can be considerable due to the extremely high column density of the gas. In order to evaluate this, we assumed that the measured SWS flux from the 0-0 S lines is the total flux emitted by the shocked cold $\mathrm{H}_{2}$ gas of the flow and that the contribution of non-thermal emission is negligible, as seen in Sect. 3.4.1. We then computed the luminosity of an LTE gas at $T=520 \mathrm{~K}$, using the $0-0 \mathrm{~S}(1)$ observed dereddened intensity to derive the absolute intensities of the remaining lines. The luminosity of the "cold" gas is $3.6 \pm 0.6 L_{\odot}$. Added to the previous estimate, this gives a total $L_{\mathrm{H}_{2}}$ for the entire flow of $8.2 \pm 0.7 L_{\odot}$.

\subsection{Mass flux, mass, and energy of the $\mathrm{H}_{2}$ jet}

The physical and kinematical parameters inferred in the previous sections allow us to evaluate important kinematical and dynamical properties of the flow. Therefore, we estimated the mass flux rate of the flow $\left(\dot{M}_{\text {out }}\right)$ from the $\mathrm{H}_{2}$ in order to compare it with the mass outflow rate and the mass accretion rate of the protostar previously obtained from $\mathrm{CO}$ observations and models (see e.g. Shepherd et al. 2000; Cesaroni et al. 2005; Lebrón et al. 2006). The value of $\dot{M}_{\text {out }}\left(\mathrm{H}_{2}\right)$ can be written as $\dot{M}_{\mathrm{k}}=2 \mu m_{\mathrm{H}} N_{\mathrm{H}_{2}} A v_{\mathrm{t}} / l_{\mathrm{t}}$, where $\mu$ is the average atomic weight, $m_{\mathrm{H}}$ the proton mass, $N_{\mathrm{H}_{2}}$ the $\mathrm{H}_{2}$ column density, A the area of the $\mathrm{H}_{2}$ knot, $v_{\mathrm{t}}$ the tangential velocity, and $l_{\mathrm{t}}$ the projected length of the knot (see e.g. Nisini et al. 2005; Podio et al. 2006; Antoniucci et al. 2007).

Using the $2.12 \mu \mathrm{m}$ column density of Table 5 , the radial velocities of Table 4, and assuming an average inclination for all the knots of $20^{\circ}$ with respect to the plane of the sky, we estimate mass fluxes between $10^{-6}$ and $10^{-8} M_{\odot} \mathrm{yr}^{-1}$ (see Table 5, Col. 7). In fact, these values represent lower limits. From the ro-vibrational analysis, the cold gas column density is two orders of magnitude higher than the warm component (e.g. 0-0 S
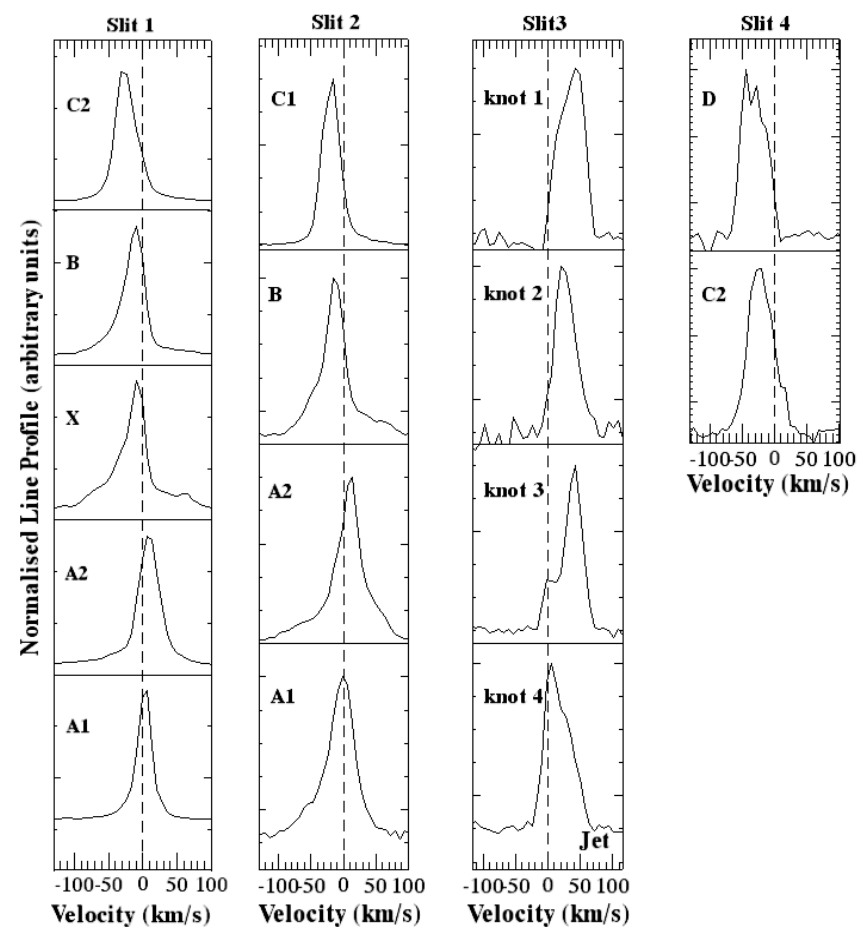

Fig. 8. Line profiles of the knots in the 1-0 S(1) line of $\mathrm{H}_{2}$ at $2.12 \mu \mathrm{m}$.

lines have a column density $N_{\mathrm{H}_{2}} \sim 9.7 \times 10^{21} \mathrm{~cm}^{-2}$ ). Using this value, and assuming the same velocity and extension of the emitting area of the $2.12 \mu \mathrm{m}$ line, we obtain $\dot{M}_{\text {out }}\left(\mathrm{H}_{2}\right) \sim$ $7.5 \times 10^{-4} M_{\odot} \mathrm{yr}^{-1}$. 

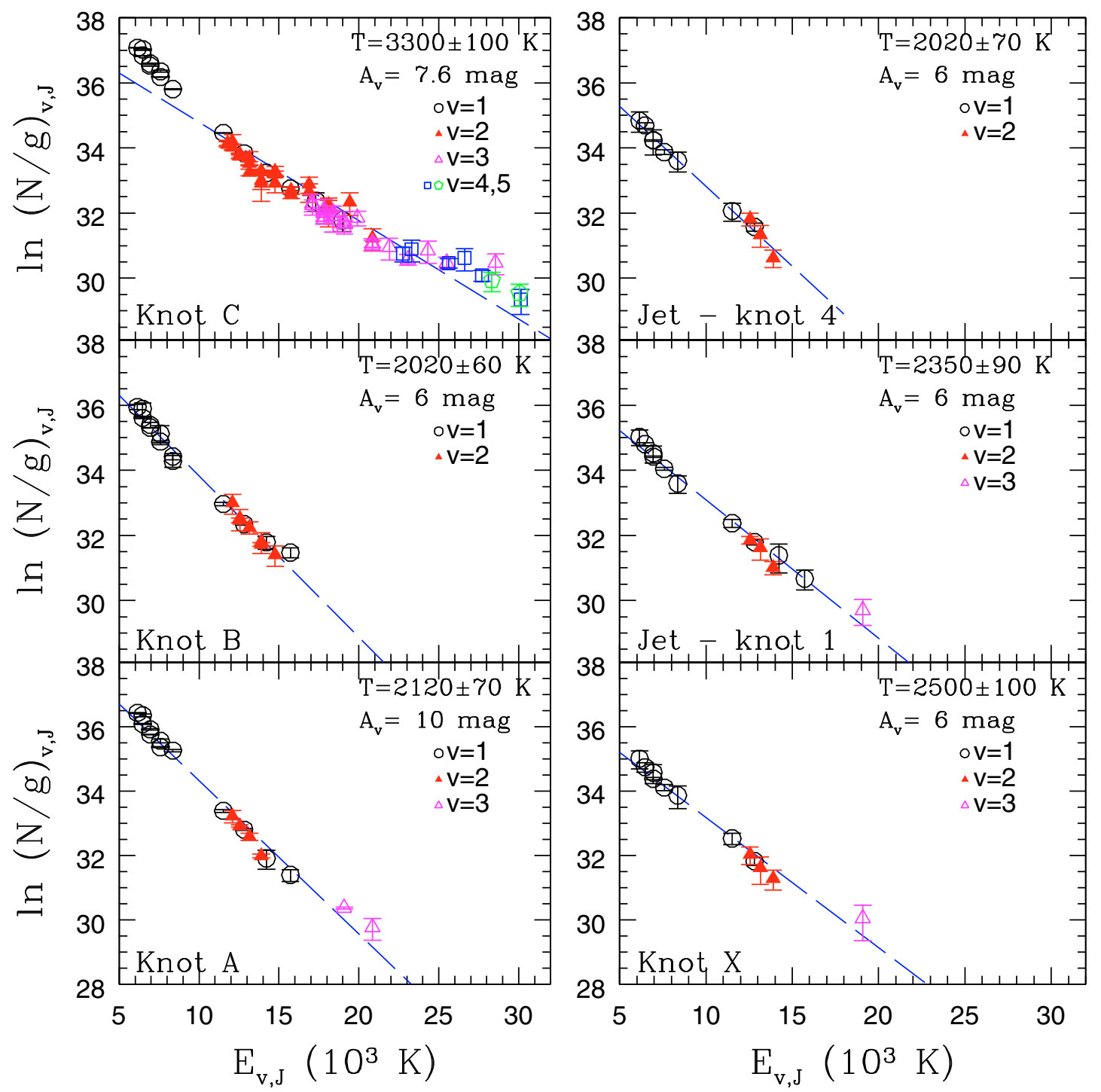

Fig. 9. Rotational diagram of knots in the IRAS 20126+4104 jet obtained from the low-resolution NIR spectroscopy. Different symbols indicate lines coming from different vibrational levels, as coded in the upper right corner of the boxes. The inferred temperature and extinction are indicated in the upper right corner of the box.

It is worth noting, however, that this value should only be considered as an estimate of the mass flux, for two reasons. Firstly, we have set $l_{\mathrm{t}}$ and $v_{\mathrm{t}}$ as equal for both $\mathrm{H}_{2}$ components. Probably, such an estimate is a lower limit, since $l_{\mathrm{t}}$ of the cold gas can be higher than the $l_{\mathrm{t}}$ measured at $2.12 \mu \mathrm{m}$. More important, the adopted mass flux formula depends on the assumption that $l_{\mathrm{t}} / v_{\mathrm{t}}$ is the cooling time $\left(t_{\mathrm{c}}\right)$ of the shock. In this case, the kinematically determined $t_{\mathrm{c}}$ of knot $\mathrm{C}$ is $1.9 \times 10^{10} \mathrm{~s}$. Also other methods have been used in the literature to derive $t_{\mathrm{c}}$. Davis et al. (2000) adopt the approximation $t_{\mathrm{c}} \sim 3 \times 10^{8} n_{6}^{-1} T_{3}^{-2.3}$ (in seconds), where $n_{6}$ is the density in units $10^{6} \mathrm{~cm}^{-3}$ and $T_{3}$ the temperature in units of $1000 \mathrm{~K}$ (Smith \& Brand 1990). In this way, adopting $n_{\mathrm{H}_{2}}=10^{5} \mathrm{~cm}^{-3}$ (Cesaroni et al. 1999) and two different temperatures, for knot $\mathrm{C}$, we would obtain $t_{\mathrm{c}}($ warm $)=$ $6.1 \times 10^{8} \mathrm{~s}$ (with $\left.T=2000 \mathrm{~K}\right)$, and $t_{\mathrm{c}}($ cold $)=1.5 \times 10^{10} \mathrm{~s}$ (with $T=500 \mathrm{~K}$ ). The $\dot{M}_{\text {out }}$ of the cold component would then be remarkably similar to the previous estimate. Moreover, it is very close to the mass outflow rates derived by Shepherd et al. (2000) and Lebrón et al. (2006) from the CO mm analysis $\left(8.1 \times 10^{-4} M_{\odot} \mathrm{yr}^{-1}\right.$, and $3.4 \times 10^{-3} M_{\odot} \mathrm{yr}^{-1}$, respectively).
In addition, we can derive a another estimate of the mass flux from the luminosity of the [O I] line (at $63 \mu \mathrm{m}$ ) using the approximate formula $\dot{M}_{\text {out }} \sim 10^{-4} \times\left(\mathrm{L}(63 \mu \mathrm{m}) / L_{\odot}\right) M_{\odot} \mathrm{yr}^{-1}$ (see e.g. Hollenbach \& McKee 1989; Liseau et al. 1997; Cabrit 2002). As a result, we get $\dot{M}_{\text {out }}(\mathrm{OI})=2 \times 10^{-4} M_{\odot} \mathrm{yr}^{-1}$. Such an estimate is an upper limit, however, since part or all of the [O I] emission could originate from the PDR.

To derive the mass of each knot, we assumed $M_{\mathrm{k}}=$ $2 \mu m_{\mathrm{H}} m_{\mathrm{H}} N_{\mathrm{H}_{2}} A$. For knot D, where $N_{\mathrm{H}_{2}}$ was not measured, we assumed an average value of $4.4 \times 10^{18} \mathrm{~cm}^{-2}$ from Table 4 . The total mass $\left(\Sigma_{k} M_{k}\right)$ of the warm gas is $\sim 10^{-3} M_{\odot}$. Assuming an average value for the inclination of the flow of $20^{\circ}$, the dynamical timescale of the flow $\left(\tau_{\mathrm{d}}\right)$ is $\sim 1.3 \times 10^{4} \mathrm{yr}$, the total momentum $P\left(\Sigma_{k} M_{k} v_{k}\right)$ is $0.06 M_{\odot} \mathrm{km} \mathrm{s}^{-1}$, the kinetic energy $E_{k}\left(\frac{1}{2} \Sigma_{k} M_{k} v_{k}^{2}\right)$ is $5 \times 10^{43} \mathrm{erg}$, and the momentum flux $\dot{P}\left(P / \tau_{d}\right)$ is $5 \times 10^{-6} M_{\odot} \mathrm{yr}^{-1} \mathrm{~km} \mathrm{~s}^{-1}$. We note that these values must be considered as lower limits, since the mass of observable shocked warm $\mathrm{H}_{2}$ is only a small fraction of the total shocked molecular hydrogen mass, as seen previously. If we compute these quantities by taking the column density of the cold component of 


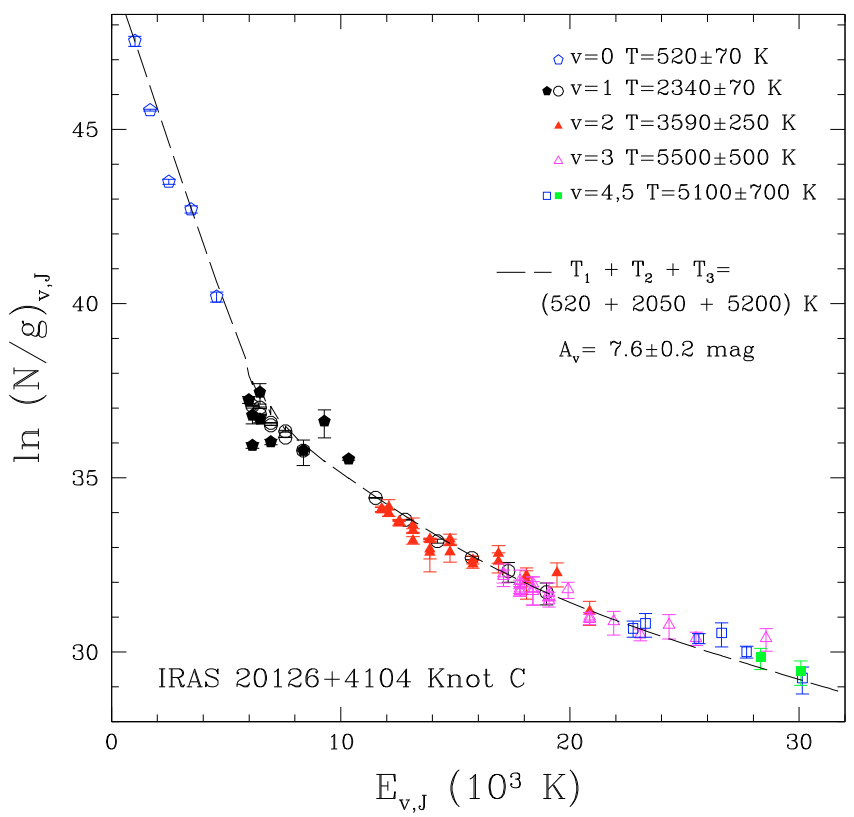

Fig. 10. Rotational diagram of knot $C$ of the IRAS 20126+4104 jet obtained from the low-resolution spectroscopy. Different symbols indicate lines coming from different vibrational levels, as coded in the upper right corner of the box $(v=0$ and $v=1$ ISO lines are indicated by open and filled pentagons, respectively). The dashed line represents the theoretical population distribution for a combination of thermalised gas at three different temperatures $(520,2050$, and $5200 \mathrm{~K}$, see text). The derived temperature from the different vibrational levels is indicated in the upper right corner of the box. The extinction is reported as well.

the gas into account, we obtain the following crude estimates: $M \sim 0.6 M_{\odot} \mathrm{km} \mathrm{s}^{-1}, P \sim 50 M_{\odot} \mathrm{km} \mathrm{s}^{-1}, E_{\mathrm{k}} \sim 4 \times 10^{46} \mathrm{erg}$, $\dot{P} \sim 4 \times 10^{-3} M_{\odot} \mathrm{yr}^{-1} \mathrm{~km} \mathrm{~s}^{-1}$.

\section{Discussion}

\subsection{A precessing jet model}

As mentioned in Sect. 3.1, the inner region of the $\mathrm{H}_{2}$ jet clearly shows a wiggling morphology, possibly indicating a small-scale precession, different from the long-period precession derived from the positions of the outer knots (Shepherd et al. 2000; Cesaroni et al. 2005).

To investigate this new morphological feature, we used a simple jet model to fit the observed position of the inner knots (see e.g. Eislöffel et al. 1996; Texeira et al. 2007):

$$
\left(\begin{array}{l}
\alpha \\
\delta
\end{array}\right)=\left(\begin{array}{c}
\alpha_{0} \\
\delta_{0}
\end{array}\right)+\left(\begin{array}{cc}
\cos (\psi) & -\sin (\psi) \\
\sin (\psi) & \cos (\psi)
\end{array}\right) \times\left(\begin{array}{c}
\phi l \sin \left(2 \pi l / \lambda+\chi_{0}\right) \\
l
\end{array}\right)
$$

where $\alpha_{0}, \delta_{0}$ are the coordinates of the source, $\psi$ the rotation angle, $\phi$ the precession amplitude (in radiants), $l$ the distance from the source position, $\lambda$ the precession length scale, $\chi_{0}$ the initial phase at the source. The resulting model, shown in Fig. 11, was been obtained for values of $\psi=-60.9, \phi=0.133(\sim 7.6)$, $\lambda \sim 10^{\prime} .9$, and $\chi_{0}=81^{\circ}$. 6 . The inclination of the flow with respect to the plane of the sky was not considered in the fit, since its value (close to the source) is relatively small $\left(\sim 9^{\circ}\right)$. For a constant jet velocity of $80 \mathrm{~km} \mathrm{~s}^{-1}$, at a distance $(D)$ of $1.7 \mathrm{kpc}$, we obtained a precession period of $\sim 1100 \mathrm{yr}$, which appears short in comparison with the value of $\sim 64000 \mathrm{yr}$ derived in Shepherd et al. (2000) and Cesaroni et al. (2005). We then tried to reproduce the complex motion of the flow, adding a second precession

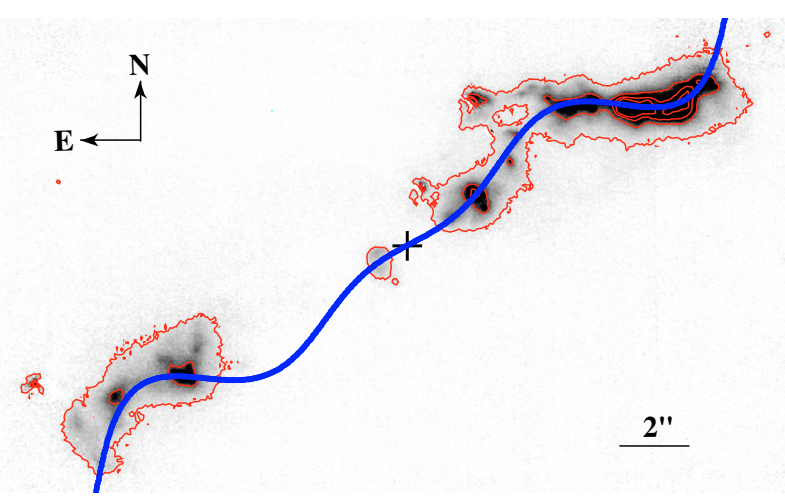

Fig. 11. Precession model plotted over the $\mathrm{H}_{2}-\mathrm{Br} \gamma$ image from Subaru. The model is for a precession angle of 7.6 and a precession scale of 10.'9. A cross indicates the position of the source.

mode to our model, that should take the longer period traced by the outer knots into account. We were, however, not able to fairly match the pattern of the entire jet.

Finally, we can also give a very rough estimate of the density for the cold component, as well. If we assumed that the size for knot $\mathrm{C}$ along the line of sight were similar to the observed length $\left(\sim 10^{17} \mathrm{~cm}\right)$, we would obtain $n_{\mathrm{H}_{2}} \sim 10^{5} \mathrm{~cm}^{-3}$, as in Cesaroni et al. (1999).

This could indicate that the origin of the two observed 'precessions' is different or that the precession period changed over time. The long-period precession was interpreted by Shepherd et al. (2000) and Cesaroni et al. (2005) as caused by the interaction between the disc of IRAS 20126+4104 and a stellar companion of a few solar masses. However, it is unlikely that the small-period precession we observe is caused by the orbital motion of the jet source around its companion. Since the orbital radius is given by the following equation (see Anglada et al. 2007) $r=\frac{\lambda \tan (\phi) D}{2 \pi}$, the resulting value is $r \sim 400 \mathrm{AU}$. The radius of IRAS $20126+4104$ disc was estimated around 800 AU (Cesaroni et al. 1997), meaning that the companion would intersect the disc. Alternatively, the precession could have changed with time. The ejection of a third companion in a hierarchical triple system, for example, might have led to the formation of a tighter binary system and to shortening of the orbital period. However, this hypothesis does not explain the different position angles on the sky measured in the two precession modes $\left(-60^{\circ} .9\right.$ and $-37^{\circ}$, respectively).

It seems more likely, then, that the complex axis-wandering is induced by tidal interactions of multiple stellar companions. Indeed IRAS 20126+4104 is not a single compact source, but is composed of a small cluster of YSOs (De Buizer 2007); therefore, the dynamics of such a system can be extremely complicated. It is indeed beyond of the scope of this paper to obtain a rigorous modelling for the dynamics of such a system.

\section{2. $\mathrm{H}_{2}$ jet vs. CO outflow}

Observations of protostellar jets in low-mass YSOs support the idea that their molecular outflows are driven and accelerated by the highly-collimated bipolar jets. Outflows from intermediateand high-mass sources, on the other hand, are often poorly collimated, and only in a few cases is there clear evidence of a driving jet. This has often led to the suggestion that these outflows could be driven by a different mechanism, such as, a wide-angle radial wind (see e.g. Arce et al. 2007). 
Table 5. Physical parameters of the $\mathrm{H}_{2}$ knots in IRAS 20126+4104 derived from the low-resolution spectroscopy and imaging.

\begin{tabular}{ccccccc}
\hline \hline Knot & $\begin{array}{c}\text { Average Temperature } \\
(\mathrm{K})\end{array}$ & $\begin{array}{c}A_{\mathrm{v}}\left(\mathrm{H}_{2}\right) \\
(\mathrm{mag})\end{array}$ & $\begin{array}{c}N_{\mathrm{H}_{2}} \\
10^{18} \mathrm{~cm}^{-2}\end{array}$ & $\begin{array}{c}\text { Flux }(2.12 \mu \mathrm{m}) \\
\left(10^{-14} \mathrm{erg} \mathrm{cm}^{-2} \mathrm{~s}^{-1}\right)\end{array}$ & $\begin{array}{c}L_{\mathrm{H}_{2}}^{a} \\
\left(L_{\odot}\right)\end{array}$ & $\begin{array}{c}\dot{M}_{\text {out }}\left(\mathrm{H}_{2}\right)^{b} \\
\left(10^{-8} M_{\odot} \mathrm{yr}^{-1}\right)\end{array}$ \\
\hline A & $2120 \pm 70$ & $10 \pm 3$ & 5.4 & $29.9 \pm 0.2$ & $0.70 \pm 0.13$ & 18 \\
B & $2020 \pm 60$ & $6 \pm 1^{c}$ & 3.6 & $10.9 \pm 0.1$ & $0.21 \pm 0.03$ & 6 \\
C & $3300 \pm 100$ & $7.6 \pm 0.2$ & 12.5 & $71.5 \pm 0.3$ & $3.12 \pm 0.24$ & 192 \\
D & $\ldots$ & $\ldots$ & $\ldots$ & $6.4 \pm 0.1$ & $0.12^{d}$ & $\cdots$ \\
X & $2500 \pm 100$ & $6 \pm 1^{e}$ & 1.9 & $2.4 \pm 0.1^{f}$ & $0.06 \pm 0.01$ & 2 \\
jet - knot 1 & $2350 \pm 90$ & $6 \pm 3$ & 1.7 & $7.5 \pm 0.2$ & $0.2 \pm 0.1$ & 8 \\
jet - knot 2 & $\ldots$ & $\ldots$ & $\ldots$ & $1.0 \pm 0.4$ & $0.02^{g}$ & $\ldots$ \\
jet - knot 3 & $\ldots$ & $\ldots$ & $\ldots$ & $2.7 \pm 0.5$ & $0.04^{h}$ & $\ldots$ \\
jet - knot 4 & $2020 \pm 70$ & $6 \pm 3$ & 1.2 & $10.0 \pm 0.2$ & $0.2 \pm 0.1$ & 8 \\
\hline
\end{tabular}

${ }^{a}$ Derived from the average temperature. The contribution from the cold component of the gas is not taken into account. The $L_{\mathrm{H}_{2}}$ from the cold component, as measured from the $0-0 \mathrm{~S}$ lines of the ISO spectrum, is $L_{\mathrm{H}_{2}}=3.6 \pm 0.4 L_{\odot}$; ${ }^{b}$ derived from the average temperature. The total $N_{\mathrm{H} 2}$ column density is measured by comparing the intrinsic (per unit mass) and observed flux of the $1-0 \mathrm{~S}(1)(2.12 \mu \mathrm{m})$ line; ${ }^{c} A_{\mathrm{v}}$ measured from the [Fe II] lines $(1.64$ and $1.26 \mu \mathrm{m})$ is $11 \pm 7 \mathrm{mag} ;{ }^{d}$ Computed assuming $T_{\mathrm{ex}}=2000 \mathrm{~K}$ and $A_{\mathrm{V}}=6 \mathrm{mag} ;{ }^{e} A_{\mathrm{V}}$ measured from the [Fe II] lines (1.64 and $1.26 \mu \mathrm{m})$ is $7 \pm 4 \mathrm{mag} ;{ }^{f} \operatorname{Flux}([\mathrm{Fe} \mathrm{II}])=3 \pm 1 \times 10^{-15} \mathrm{erg} \mathrm{cm}{ }^{-2} \mathrm{~s}^{-1} ;{ }^{g}$ Computed assuming $T_{\mathrm{ex}}=2350 \mathrm{~K}$ and $A_{\mathrm{v}}=6$ mag; ${ }^{h}$ Computed assuming $T_{\mathrm{ex}}=2020 \mathrm{~K}$ and $A_{\mathrm{v}}=6 \mathrm{mag}$.

Table 6. Comparison between $\mathrm{H}_{2}$ jet and $\mathrm{CO}$ outflow physical properties of the IRAS $20126+4104$ flow.

\begin{tabular}{cccc}
\hline \hline Parameter & $\mathrm{H}_{2}$ & $\mathrm{CO}^{a}$ & $\mathrm{CO}^{b}$ \\
\hline Mass $\left(M_{\odot}\right)$ & 0.6 & 53 & 16.3 \\
$v_{\text {red }}\left(\mathrm{km} \mathrm{s}^{-1}\right)$ & $(-8,47)$ & $\ldots$ & $(4,55)$ \\
$v_{\text {blue }}\left(\mathrm{km} \mathrm{s}^{-1}\right)$ & $(-14,-42)$ & $\ldots$ & $(-11,-58)$ \\
$\tau_{\mathrm{d}}\left(10^{4} \mathrm{yr}\right)$ & 1.3 & 6.4 & $\ldots$ \\
$\dot{M}\left(10^{-3} M_{\odot} \mathrm{yr}\right)$ & 0.75 & 0.81 & 3.4 \\
$E_{\mathrm{k}}\left(10^{46} \mathrm{ergs}\right)$ & 4 & 5.1 & 130 \\
$\mathrm{P}\left(M_{\odot} \mathrm{km} \mathrm{s}^{-1}\right)$ & 50 & 403 & 1490 \\
$\dot{P}\left(10^{-3} M_{\odot} \mathrm{yr}^{-1} \mathrm{~km} \mathrm{~s}^{-1}\right)$ & 4 & 6 & 310 \\
\hline
\end{tabular}

${ }^{a}$ From Shepherd et al. (2000). ${ }^{b}$ From Lebrón et al. (2006).

Apparently, IRAS 20126+4104 represents a particular example of poorly collimated outflow powered by a protostellar jet. The poor collimation of this outflow has been explained by the severe precession of the jet. It is thus interesting to investigate whether the properties of the IRAS $20126+4104 \mathrm{H}_{2}$ jet meet the physical parameters of the $\mathrm{CO}$ outflow, i.e. if the jet is powerful enough to accelerate the surrounding medium and drive the outflow. This is indeed a fundamental question, because the YSO accretion rate is often estimated from the mass flux rate of the outflow. When comparing $\mathrm{CO}$ and $\mathrm{H}_{2}$ parameters, however, we have to keep in mind that the $\mathrm{CO}$ lines only give a timeintegrated response over the lifetime of the jet, whereas the $\mathrm{H}_{2}$ emission provides an "instantaneous" measure of these quantities.

Large-scale outflow properties from $\mathrm{CO} \mathrm{mm}$ observations were extensively studied by several authors (see e.g. Shepherd et al. 2000; Lebrón et al. 2006). Since their computations are based on different assumptions, however, the results of Lebrón et al. (2006) are at least one order of magnitude larger than those of Shepherd et al. (2000).

In Table 6 we compare our $\mathrm{H}_{2}$ flow parameters (obtained including the cold component) with the parameters of the $\mathrm{CO}$ outflow from Shepherd et al. (2000) and Lebrón et al. (2006). From this table, we can infer that the jet is driving the outflow, at least partially. The radial velocities of $\mathrm{H}_{2}$ are consistent with the $\mathrm{CO}$ velocities, including the high-velocity component up to the $60 \mathrm{~km} \mathrm{~s}^{-1}$ detected by Lebrón et al. (2006). On the other hand, the total mass of the $\mathrm{H}_{2}$ bullets is just a fraction of the total $\mathrm{CO}$ mass. As a consequence, the momentum $(P)$ of the jet is at least one order of magnitude less than that of the outflow. However, our estimates of the mass flux, kinetic energy, and momentum flux are, indeed, almost coincident with Shepherd et al. (2000), supporting the hypothesis that the $\mathrm{H}_{2}$ jet is fully driving the flow. In Contrast, the values from Lebrón et al. (2006) are from one to two orders of magnitude more than ours (see Table 6). This would imply that the $\mathrm{H}_{2}$ jet is only partially driving the outflow and that there is an undetected component along the flow, likely a neutral jet, that is supplying further momentum flux to the outflow. However, it is worth noting that the mass flux obtained by Lebrón et al. (2006) is slightly higher than the mass accretion rate of IRAS 20126+4104 inferred by Cesaroni et al. (2005) $\left(2 \times 10^{-3} M_{\odot} \mathrm{yr}^{-1}\right)$, and this is quite unlikely. This discrepancy could be then explained by the presence of (undetected) multiple jets (winds) driven by other YSOs near the source that would substantially contribute to power the $\mathrm{CO}$ outflow.

\subsection{Shock conditions along the jet}

As the previous results point out, the cold $\mathrm{H}_{2}$ component of the jet plays a major role in the kinematics and dynamics of this outflow. It is therefore desirable to discuss the origin of this component in more detail.

A prevalence of C-type shocks along the flow could explain our findings. Indeed C-type shocks produce a high column density in the 0-0 lines (see e.g. Flower et al. 2003; McCoey et al. 2004), which are very sensitive to these shocks. The column densities in our $0-0$ lines could not be explained by a Jtype shock (see e.g. Le Bourlot et al. 2002). Except for the [Fe II] and the [OI] emission close to the source, the emission observed along the flow only comes from the $\mathrm{H}_{2}$. Only the inner region near the source position exhibits hints of ionic emission. There is no evidence of the ionised jet detected in the radio by Hofner et al. (2007), probably confined to a region very close to the source and highly extincted. On the other hand, the complete absence of any ionic emission in the other knots of the flow should not be attributable to a high extinction value, since the measured $A_{\mathrm{v}}$ values along the jet are relatively low. Such a behaviour has already been observed in several lowmass protostellar jets. They usually show only $\mathrm{H}_{2}$ emission, or, at most, a hint of ionic emission close to the exciting source (see e.g. Giannini et al. 2004; Caratti o Garatti et al. 2006). It is also clearly observed in the high-mass IRAS 18151-1208 jet 
spectra (Davis et al. 2004). They are interpreted as C-type shocks or as J-shocks with magnetic precursors, which are J-type shock waves evolving into "continuous" C-type shock waves (see e.g. Giannini et al. 2004). Our kinematical observations also seem to indicate the presence of C-type shocks. The $\mathrm{H}_{2}$ velocities in C-type shocks can exceed $50-60 \mathrm{~km} \mathrm{~s}^{-1}$ up to $80 \mathrm{~km} \mathrm{~s}^{-1}$ (see e.g. Le Bourlot et al. 2002). Accordingly, the $F W Z I$ of $\mathrm{H}_{2}$ bow shocks can be up to twice this value, depending on the inclination of the jet axis with respect to the plane of the sky (see e.g. Davis et al. 2004), i.e. the broadest lines are expected for a bow shock located in the plane of the sky. Actually, if we consider the different inclinations for the inner and the outer knots, our observations seem to indicate a high shock velocity in both regions, whereas the higher value of the FWZI is observed in the inner knots (closer to the sky plane), and a narrower shape in the outer knots. From these values we can roughly estimate a shock velocity between 40 and $80 \mathrm{~km} \mathrm{~s}^{-1}$, where the fastest speed would be attained in the inner region. Such high shock velocities (50-80 $\mathrm{km} \mathrm{s}^{-1}$ ) imply, however, low pre-shock densities of $10^{3}-10^{4} \mathrm{~cm}^{-3}$ (Le Bourlot et al. 2002) in C shocks, assuming a magnetic field $B(\mu \mathrm{G})=b\left[n_{H}\left(\mathrm{~cm}^{-3}\right)\right]^{-0.5}$, with $b=1$. Higher densities lead to a lower maximum shock speed $\left(v_{\text {diss }}\right)$, which is the maximum shock velocity that can be attained prior to the collisional dissociation of $\mathrm{H}_{2}$. In contrast, both $\mathrm{CO}$ and $\mathrm{H}_{2}$ observations seem to indicate a higher pre-shock density $\left(10^{5}-10^{6} \mathrm{~cm}^{-3}\right)$ in the medium (see e.g. Cesaroni et al. 1999). In this case, $v_{\text {diss }}$ would decrease to $30-50 \mathrm{~km} \mathrm{~s}^{-1}$ (Le Bourlot et al. 2002). Thus a "standard" C-type model cannot entirely explain our findings close to the source. A larger transversal magnetic field $(b>1)$ or a different shock model (such as a J-shock with a magnetic precursor) is probably needed. This last hypothesis would also explain the presence of the ionic emission in the knots close to the source.

\section{4. $L_{\mathrm{H}_{2}}$, accretion and ejection rates}

Several authors have argued that a tight correlation between the evolutionary properties of YSOs and their outflows exists. This is particularly true for those objects forming by disc accretion, since accretion and ejection should be regulated by the same mechanism. Recently, it has been shown for low-mass YSOs that the total $\mathrm{H}_{2}$ luminosity of the jets is proportional to their accretion rates (see e.g., Smith 2002; Froebrich et al. 2003). In particular, an empirical relationship between $L_{\mathrm{H}_{2}}$ and $L_{\mathrm{bol}}$ $\left(L_{\mathrm{H}_{2}} \propto L_{\text {bol }}^{0.58}\right.$, Caratti o Garatti et al. 2006) was derived for a large sample of low-mass protostellar jets. This correlation holds for very young YSOs (Class 0 and some Class I), where the bolometric luminosity is mostly coincident with the accretion luminosity of the object and implies that $\dot{M}_{\text {acc }}$ increases with the luminosity (i.e. mass) of the protostar. In Fig. 12 we report the results obtained from the sample of Caratti o Garatti et al. (2006). The diagram compares the measured outflow $\mathrm{H}_{2}$ luminosity versus the bolometric source luminosity, both on a logarithmic scale. We have included the new data from IRAS 20126+4104 (positioned in the upper right corner). The best fit previously obtained fits our object, perfectly, suggesting that the relationship also applies to more massive jets in their earliest stage of formation. According to the dynamical timescales of the outflow and the jet (a few times $10^{4}$ years), IRAS 20126+4104 has not yet reached the main sequence (MS) and has not yet developed any hypercompact HII (HCHII) region, which may affect the collimation of the jet/outflow system (Beuther \& Shepherd 2005). Most importantly, the bolometric luminosity of the source mainly comes from accretion (see Cesaroni et al. 1999, 2005). In these works, the authors obtain for this source an

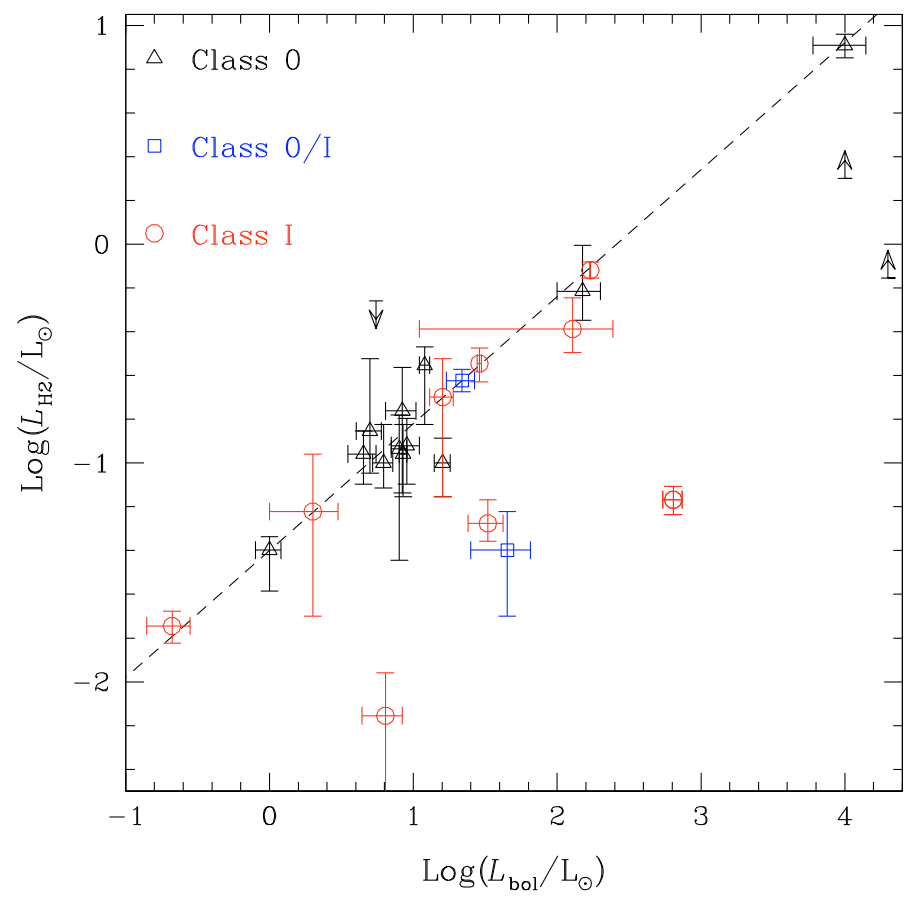

Fig. 12. $L_{\mathrm{H}_{2}}$ vs. $L_{\text {bol }}$ including the IRAS $20126+4104$ jet (also considering the luminosity of the cold component, the new datapoint is located in the upper-right corner of the diagram). Values for IRAS 18151-1208 and IRAS 11101-5829 jets have been included as lower limits, as well (see discussion in the text). A dashed line indicates the previous fit from Caratti o Garatti et al. (2006).

accretion luminosity of $1.2 \times 10^{4} L_{\odot}$ for a mass accretion rate of $\sim 1-2 \times 10^{-3} M_{\odot} \mathrm{yr}^{-1}$. Moreover, that we obtain an ejection rate of $30-40 \%$ for the accretion rate from the $\mathrm{H}_{2}$ strengthens the reliability of our findings, since for high-mass sources we would expect a ratio of the mass ejection/accretion rate higher than the value $(\sim 0.1)$ usually derived for the low-mass objects (see e.g. Cabrit 2007).

Finally, we compared our results with the $L_{\mathrm{H}_{2}}$ of two highmass jets previously investigated by means of NIR spectroscopy, i.e. IRAS 18151-1208 $\left(L_{\mathrm{H}_{2}}=0.7 L_{\odot}\right.$, Davis et al. 2004) and IRAS 11101-5829 $\left(L_{\mathrm{H}_{2}} \geq 2 L_{\odot}\right.$, Gredel 2006). These sources have almost the same $L_{\text {bol }}$ as IRAS 20126+4104. The $L_{\mathrm{H}_{2}}$ estimates are quite close to the $\mathrm{H}_{2}$ luminosity of IRAS $20126+4104$ obtained from the warm $\mathrm{H}_{2}$ component (i.e. from our NIR analysis, $L_{\mathrm{H}_{2}}=4.6 \pm 0.3 L_{\odot}$ ). For comparison, the values of the IRAS 18151-1208 and IRAS 11101-5829 jets were also included as lower limits in Fig. 12. It is also worth noting that the slightly lower value found by Davis et al. (2004) could be caused by the high extinction values (10-30 mag) observed towards this flow. Indeed an MIR investigation could reveal whether the cold $\mathrm{H}_{2}$ component plays a major role in the cooling of those jets, as well.

\subsection{Comparing high- and low-mass jets}

The three high-mass protostellar jets spectroscopically investigated up to date share similar characteristics. Like the low-mass jets, they are collimated and powerful enough to drive their outflows. The jet is formed close to the source, as we observe e.g. in IRAS 20126+4104 knot X (located $\sim 1700$ AU from the source) or in IRAS 11101-5829 knot HH136 J2 (located 3000 AU from the source), indicating that the collimation of the jet occurs close to the source. They show molecular and ionic shocked emission 
along the flow, and no evidence of fluorescent excitation. The observed jet velocities are similar to those of the CO outflow. Where the extinction is low, some jets (e.g. IRAS 11101-5829, IRAS 18162-2048) also show HH objects. Some kinematical and dynamical quantities, such as mass flux, momentum flux, luminosity, and kinetic energy, are greater, however, than in low-mass jets, because the powering YSO is more massive. Moreover, a precession-wiggling morphology is observed in most of the massive collimated $\mathrm{H}_{2}$ jets, often with large precessing angles (up to $\sim 40^{\circ}$ in IRAS 20126+4104) (e.g. IRAS 16547-4247, Brooks et al. 2003; IRAS 18151-1208, Davis et al. 2004; IRAS 111015829, Gredel 2006; M17 disc silhouette, Nürnberger et al. 2007; IRAS 07427-2400, IRAS 20293+3952, and IRAS 23033+5951, Nanda Kumar et al. 2002). Precession is also observed in lowmass jets, but it is neither as frequent nor as pronounced (usually the precession angles are less than $10^{\circ}$ ). All this indicates that the dynamical interactions among massive stars are stronger and more frequent than in low-mass star-forming regions. Such complex dynamics could also explain the confused $\mathrm{H}_{2}$ morphology of some jets and the lack of collimation in some massive outflows. Finally, the lack of jets and the poor outflow collimation observed in several massive young sources fits the evolutionary outflow scenario proposed by Beuther \& Shepherd (2005) well. In this context, those sources that have jets detected toward them are very young (well before the MS turn-on), while those without detectable jets near the protostar have ultracompact HII (UCHII) regions. If the disappearance of a collimated jet in early B protostars stems from the presence of enhanced ionising radiation from an accreting early main sequence star, then all early B stars may be formed via accretion. In this sense, they are scaledup versions of low-mass protostars at early phases (such as for IRAS 20126+4104, IRAS 18151-1208, IRAS 11101-5829, etc.). As YSOs evolve, developing UCHII regions that destroy the disc, the jets finally disappear, and the outflows look more like poorly collimated wind-blow bubbles (see also Arce et al. 2007).

In conclusion, the three high-mass protostellar jets spectroscopically studied appear to be scaled-up versions of the lowmass ones. Furthermore, a morphological analysis of the few intermediate- high-mass $\mathrm{H}_{2}$ jets known up to now partially supports an evolutionary jet/outflow scenario. A larger sample of intermediate-/high-mass jets is, however, needed, and it would be premature to jump to the conclusion that the disc accretionejection paradigm can be extended to the intermediate- and highmass protostars.

\section{Conclusions}

The IRAS $20126+4104 \mathrm{H}_{2}$ jet has been extensively investigated through near-IR $\mathrm{H}_{2}$ and [Fe II] narrow-band imaging, $\mathrm{H}_{2}$ highresolution spectroscopy, along with low-resolution spectroscopy $(0.9-200 \mu \mathrm{m})$ throughout the infrared wavelength range. The kinematical, dynamical, and physical conditions of the $\mathrm{H}_{2}$ gas along the flow were probed. The main results of this work are the following:

- A high angular resolution $\mathrm{H}_{2}$ continuum-subtracted image from Subaru reveals a small-scale precession of the jet close to the source, with an angle of $(\sim 7.6)$ and a period of $\sim 1100 \mathrm{yr}$. This is about a factor 50 shorter than the precession period deduced from large-scale $\mathrm{H}_{2}$ images.

- $\mathrm{H}_{2}$ and [Fe II] narrow-band images show the appearance of a new knot, labelled X, roughly $1^{\prime \prime}$ from the source position. No further ionic emission is detected along the flow in the narrow-band imaging, indicating that the jet is mainly molecular.
- Low-resolution spectra are rich in $\mathrm{H}_{2}$ emission, and no ionic emission is detected along the flow, with the exception of faint emission of [Fe II] close to the source position (in knots $\mathrm{X}$ and B). Faint [O I] and [C II] emissions are observed in the ISO-LWS spectrum. They could arise from an embedded PDR region around the source.

- The peak radial velocities of the knots range from -42 to $-14 \mathrm{~km} \mathrm{~s}^{-1}$ in the blue lobe and from -8 to $47 \mathrm{~km} \mathrm{~s}^{-1}$ in the red lobe. Their line profiles, very broad and often with two or three velocity components, seem to indicate a bow shock structure. In both lobes, the absolute peak radial velocities of knots close to the source (A...C) are slower $\left(0-30 \mathrm{~km} \mathrm{~s}^{-1}\right)$ than those located at greater distances (i.e. knot D, and knots 1 to $4,40-50 \mathrm{~km} \mathrm{~s}^{-1}$ ). This possibly confirms a change in the flow inclination angle (with respect to the sky) from $\sim 9^{\circ}$ (close to the source), to $\sim 45^{\circ}$ (in the outer knots). Assuming these inclination values, the spatial velocity of the knots is between 50 and $80 \mathrm{~km} \mathrm{~s}^{-1}$.

- The ro-vibrational diagrams indicate $\mathrm{H}_{2}$ excitation temperatures between 2000 and $2500 \mathrm{~K}$. Stratification of temperature is detected only in knot $\mathrm{C}$, which can be modelled combining a warm $\left(T_{\mathrm{ex}}=2050 \mathrm{~K}\right)$ and a hot $(\sim 5200 \mathrm{~K}) \mathrm{H}_{2}$ component. Additionally, the ISO-SWS spectrum reveals the presence of a cold component $(520 \mathrm{~K})$ with a high column density.

- Furthermore, our analysis seems to indicate that the $\mathrm{H}_{2}$ is mostly excited in C-type shocks, and no evidence of fluorescent excitation has been observed.

- The estimated $L_{\mathrm{H}_{2}}$ is $8.1 \pm 0.7 L_{\odot}$, where the cold component contributes about $50 \%$ to the whole radiative cooling. The high $\mathrm{H}_{2}$ luminosity suggests that IRAS 20126+4104 has a significantly increased accretion rate compared to the lowmass YSOs. This is also supported by the measured mass flux rates from $\mathrm{H}_{2}$ lines $\left(\dot{M}_{\text {out }}\left(\mathrm{H}_{2}\right) \sim 7.5 \times 10^{-4} M_{\odot} \mathrm{yr}^{-1}\right)$ matching the previous $\mathrm{CO}$ estimates. Our analysis also indicates that the cold $\mathrm{H}_{2}$ component plays a major role in the kinematics and dynamics of this flow.

- Comparing the $\mathrm{H}_{2}$ and outflow parameters strongly indicates that the jet is driving the outflow, at least partially.

- By comparing the measured luminosity of the $\mathrm{H}_{2}$ jet with the source bolometric luminosity (assumed representative of the accretion luminosity), we show that IRAS 20126+4104 fits the correlation well between these two quantities already found for low-mass protostellar jets (Caratti o Garatti et al. 2006).

- Considering our results and the literature data of a few intermediate- and high-mass protostellar jets, we conclude that these few jets appear to be scaled-up versions of their low-mass protostellar counterparts.

Acknowledgements. We would like to thank the referee, Debra Shepherd, for her helpful suggestions, which improved the manuscript.

The present work was supported in part by the European Community's Marie Curie Actions-Human Resource and Mobility within the JETSET (Jet Simulations, Experiments and Theory) network under contract MRTN-CT-2004 005592. Our observations have been funded by the Optical Infrared Coordination network (OPTICON), a major international collaboration supported by the Research Infrastructures Programme of the European Commission's Sixth Framework Programme.

The version of the ISO data presented in this paper corresponds to the Highly Processed Data Product (HPDP) set called 35500738 by W. Frieswijk et al. and HPDP set called 04300333 by Lloyd, Lerate, \& Grundy, available for public use in the ISO Data Archive.

The United Kingdom Infrared Telescope is operated by the Joint Astronomy Centre on behalf of the Science and Technology Facilities Council of the United Kingdom.

Based in part on observations made with the Italian Telescopio Nazionale Galileo (TNG) operated on the island of La Palma by the Fundación Galileo Galilei 
of the INAF (Istituto Nazionale di Astrofisica) at the Spanish Observatorio del Roque de los Muchachos of the Instituto de Astrofisica de Canarias.

Based in part on data collected at Subaru Telescope and obtained from the SMOKA, which is operated by the Astronomy Data Center, National Astronomical Observatory of Japan.

This research has also made use of NASA's Astrophysics Data System Bibliographic Services and the SIMBAD database, operated at CDS, Strasbourg, France, and the 2MASS data, obtained as part of the Two Micron All Sky Survey, a joint project of the University of Massachusetts and the Infrared Processing and Analysis Center/California Institute of Technology, funded by the National Aeronautics and Space Administration and the National Science Foundation.

\section{References}

Anglada, G., López, R., Estalella, R., et al. 2007, AJ, 133, 2799 Antoniucci, S., Nisini, B., Giannini, T., \& Lorenzetti, D. 2008, A\&A, 479, 503 Arce, H. G., Shepherd, D., Gueth, F., et al. 2007, in Protostars and Planets V, ed. B. Reipurth, D. Jewitt, \& K. Keil, 245

Ayala, S., Curiel, S., Raga, A. C., et al. 1998, A\&A, 332, 1055

Baba, H., Yasuda, N., Ichikawa, S., et al. 2002, ADASS XI, ed. D. A. Bohlender, D. Durand, \& T. H. Handley, ASP Conf. Ser., 281, 298

Baffa, C., Comoretto, G., Gennari, S., et al. 2001, A\&A, 378, 722

Beuther, H., \& Shepherd, D. 2005, in Cores to Clusters: Star Formation with Next Generation Telescopes, ed. M. S. N. Kumar, M. Tafalla, \& P. Caselli, 105

Black, J. H., \& van Dishoeck, E. 1987, ApJ, 322, 412

Bragg, S. L., Smith, W. H., \& Brault, J. W. 1982, ApJ, 263, 999

Brooks, K. J., Garay, G., Mardones, D., \& Bronfman, L. 2003, ApJ, 594, L131 Burton, M. 1992, AuJPh 45, 463

Cabrit, S. 2002, in Star Formation and the Physics of Young Stars, ed. J. Bouvier, \& J.-P. Zahn, EAS Publ. Ser., 3, 147

Cabrit, S. 2007, in Jets from Young Stars, Lect. Notes Phys. (Berlin, Heidelberg: Springer-Verlag), 723

Camenzind, M. 1990, RvMA 3, 234

Caratti o Garatti, A., Giannini, T., Nisini, B., \& Lorenzetti, D. 2006, A\&A, 449, 1077

Cesaroni, R., Felli, M., Testi, L., et al. 1997, A\&A, 325, 725

Cesaroni, R., Felli, M., Jenness, T., et al. 1999, A\&A, 345, 949

Cesaroni, R., Neri, R., Olmi, L., et al. 2005, A\&A, 434, 1039

Clegg, P. E., Ade, P. A. R., Armand, C., et al. 1996, A\&A, 315, L38

Davis, C. J., Berndsen, A., Smith, M. D., et al. 2000, MNRAS, 314, 241

Davis, C. J., Hodapp, K. W., \& Desroches, L. 2001, A\&A, 377, 285

Davis, C. J., Varricatt, W. P., Todd, S. P., \& Ramasy Howat, S. K. 2004, A\&A, 425,981

Dabroski, I. 1984, Can. J. Phys., 62, 1634

de Graauw, T., Haser L. N., Beintema, D. A., et al. 1996, A\&A, 315, L49

De Buizer, J. M. 2007, ApJ, 654, L147

Eislöffel, J., Smith, M. D., Davis, C. J., \& Ray, T. P. 1996, AJ, 112, 2086

Flower, D. R., Le Bourlot, J., Pineau des Forêts, G., \& Cabrit, S. 2003, MNRAS 341,70
Froebrich, D., Smith, M. D., \& Eislöffel, J. 2002, A\&A, 385, 239

Froebrich, D., Smith, M. D., Hodapp, K. W., \& Eislöffel, J. 2003, MNRAS, 346, 163

Giannini, T., Nisini, B., Caratti o Garatti, A., \& Lorenzetti, D. 2002, ApJ, 570, L33

Giannini, T., McCoey, C., Caratti o Garatti, A., et al. 2004, A\&A, 419, 999

Giannini, T., McCoey, C., Nisini, B., et al. 2006, 459, 821

Gredel, R. 2006, A\&A, 457, 157

Gredel, R. 2007, A\&A, 474, 941

Hofner, P., Cesaroni, R., Olmi, L., et al. 2007, A\&A, 465, 197

Hollenbach, D., \& McKee, C. F. 1989, ApJ, 342, 306

Hora, J. L., \& Latter, W. B. 1994, ApJ, 437, 281

Hunt, L. K., Mannucci, F., Testi, L., et al. 1998, AJ, 115, 2594

Kaufman, M. J., Wolfire, M. G., Hollenbach, D., \& Luhman, M. L. 1999, ApJ, 527,795

Le Bourlot, J., Pineau des Forêts, G., Flower, D. R., \& Cabrit, S. 2002, MNRAS, 332,985

Lebrón, M., Beuther, H., Schilke, P., \& Stanke, Th. 2006, A\&A, 448, 1037

Liseau, R., Giannini, T., Nisini, B., et al. 1997, in Herbig-Haro Flows and the Birth of Low Mass Stars, ed. B. Reipurth, \& C. Bertout, 111

Martí, J., Rodríguez, L. F., \& Reipurth, B. 1995, ApJ, 449, 184

McCoey, C., Giannini, T., Flower, D. R., \& Caratti o Garatti, A. 2004, MNRAS, 353,813

Mountain, C. M., Robertson, D., Lee, T. J., \& Wade, R. 1990, in Proc. SPIE, 1235,25

Murakawa, K., Suto, H., Tamura, M., et al. 2003, in Proc. SPIE, 4841, 881

Kumar, M. S. Nanda, Bachiller, R., \& Davis, C. J. 2002, ApJ, 576, 313

Nisini, B., Caratti o Garatti, A., Giannini, T., \& Lorenzetti, D. 2002, A\&A, 393, 1035

Nisini, B., Bacciotti, F., Giannini, T., et al. 2005, A\&A, 441, 159

Nürnberger, D. E. A., Chini, R., Eisenhauer, F., et al. 2007, A\&A, 465, 931

Podio, L., Bacciotti, F., Nisini, B., et al. 2006, A\&A, 456, 189

Pudritz, R. E., \& Norman, C. A. 1986, AJ, 301, 571

Ramsay Howat, S. K., Todd, S., Leggett, S., et al. 2004, in Proc. SPIE, 5492, 1160

Rieke, G. H., \& Lebofsky, M. J. 1985, ApJ, 288, 618

Rousselot, P., Lidman, C., Cuby, J-G., Moreels, G., \& Monnet, G. 2000, A\&A, 354,1134

Schultz, A. S. B., Burton, M. G., \& Brand, P. W. J. L. 2005, MNRAS, 358, 1195

Shepherd, D. S. 2003, in Massive Star Birth: A Crossroads of Astrophysics, ed. R. Cesaroni, M. Felli, E. Churchwell, \& M. Walmsley (Cambridge: Cambridge University Press), Proc. IAU Symp., 227, 237

Shepherd, D. S., Yu, K. C., Bally, J., \& Testi, L. 2000, ApJ, 535, 883

Smith, M. D. 2002, in The Origins of Stars and PLanets; the VLT View, ESO

Astrophysics Symposia, ed. J. Alves, \& M. McCaughrean

Smith, M. D., \& Brand, P. W. J. L. 1990, MNRAS, 314, 108

Sridharan, T. K., Williams, S. J., \& Fuller, G. A. 2005, ApJ, 631, L73

Su, Y.-N., Liu, S.-Y., Chen, H.-R., et al. 2007, ApJ, 671, 571

Teixeira, P. S., McCoey, C., Fich, M., \& Lada, C. J. 2007, MNRAS, in press

Wolniewicz, L., Simbiotin, J., \& Dalgarno, A. 1998, ApJS, 115, 293

Zinnecker, H., \& Yorke, H. W. 2007, ARA\&A, 45, 481 